\title{
From proteomics to discovery of first-in- class ST2 inhibitors active in vivo
}

\author{
Abdulraouf M. Ramadan, ${ }^{1}$ Etienne Daguindau, ${ }^{1}$ Jason C. Rech, ${ }^{2}$ Krishnapriya Chinnaswamy, ${ }^{3}$ \\ Jilu Zhang, ${ }^{1}$ Greg L. Hura, ${ }^{4,5}$ Brad Griesenauer, ${ }^{1}$ Zachary Bolten, ${ }^{1}$ Aaron Robida, ${ }^{3}$ Martha Larsen, ${ }^{3}$ \\ Jeanne A. Stuckey, ${ }^{3,6}$ Chao-Yie Yang, ${ }^{2}$ and Sophie Paczesny ${ }^{1}$ \\ 'Department of Pediatrics and Immunology, Indiana University School of Medicine, Indianapolis, Indiana, USA. \\ ${ }^{2}$ Department of Internal Medicine, Hematology and Oncology Division, University of Michigan, Ann Arbor, Michigan, USA \\ ${ }^{3}$ Life Sciences Institute, University of Michigan, Ann Arbor, Michigan, USA. "4awrence Berkeley National Laboratory, \\ Berkeley, California, USA. ${ }^{5}$ Department of Chemistry and Biochemistry, University of California, Santa Cruz, Santa Cruz, \\ California, USA. ${ }^{6}$ Department of Biological Chemistry, University of Michigan, Ann Arbor, Michigan, USA.
}

Soluble cytokine receptors function as decoy receptors to attenuate cytokine-mediated signaling and modulate downstream cellular responses. Dysregulated overproduction of soluble receptors can be pathological, such as soluble ST2 (sST2), a prognostic biomarker in cardiovascular diseases, ulcerative colitis, and graft-versus-host disease (GVHD). Although intervention using an ST2 antibody improves survival in murine GVHD models, SST2 is a challenging target for drug development because it binds to IL-33 via an extensive interaction interface. Here, we report the discovery of small-molecule ST2 inhibitors through a combination of high-throughput screening and computational analysis. After in vitro and in vivo toxicity assessment, 3 compounds were selected for evaluation in 2 experimental CVHD models. We show that the most effective compound, iST2-1, reduces plasma sST2 levels, alleviates disease symptoms, improves survival, and maintains graft-versus-leukemia activity. Our data suggest that iST2-1 warrants further optimization to develop treatment for inflammatory diseases mediated by SST2.

Conflict of interest: SP has a patent (US 20130115232A1, WO 2013066369A3) on "Methods of detection of graft-versus-host disease" licensed to Viracor-IBT laboratories.

Submitted: December 11, 2017 Accepted: June 12, 2018 Published: July 25, 2018

\section{Reference information:} JCI Insight. 2018;3(14):e99208. https://doi.org/10.1172/jici. insight.99208.

\section{Introduction}

Secreted cytokines transmit messages between cells to initiate defensive immune responses and to promote tissue homeostasis during immune resolution $(1,2)$. These extracellular cytokines can be regulated by clearance or can be inactivated via sequestration by soluble cytokine receptors (3). Circulatory soluble cytokine receptors are produced by a number of mechanisms, including proteolytic shedding of the membrane cytokine receptors and alternative gene splicing (4). Soluble cytokine receptors retain the abilities to recognize and bind their corresponding cytokines, similarly to their membrane-bound forms, and function as decoy receptors to attenuate cytokine concentrations. Modification of the cytokine-mediated immune response by soluble cytokine receptors provides feedback control of cytokine activities.

Dysregulated production of soluble cytokine receptors to overtly dampen cytokine activities has been observed in diseases $(5,6)$. In recent serum proteomic studies, soluble ST2 (sST2) was identified as a prognostic biomarker found to be elevated in patients with heart failure and cardiovascular-related death (7-9), acute cardiac allograft rejection (10), inflammatory bowel disease $(8,11)$, and graft-versus-host disease (GVHD) (12-17). Among patients receiving allogeneic hematopoietic cell transplantation (HCT), elevated sST2 levels in patients with steroid-refractory GVHD is the factor most significantly associated with their subsequent non-relapserelated death (12). This observation has been validated across multiple transplant platforms $(12,18-20)$.

sST2 is the soluble form of the membrane-bound ST2 (hereafter referred to as ST2) (21) encoded by interleukin-1 receptor-like 1 (IL1RL1) $(22,23)$. Both sST2 and ST2 share the same extracellular domain (ECD) $(22,24)$ that bind with the only known ligand, IL-33 (21). ST2 is expressed mainly on hematopoietic cells including T helper type 2 (Th2) cells, group 2 innate lymphoid cells (ILC2), regulatory T cells (Tregs), and mast cells $(25,26)$. On ST2 ${ }^{+} \mathrm{CD} 4^{+} \mathrm{T}$ cells, ligation of IL-33 with ST2 recruits IL-1R accessory protein (IL-1RAcP) and activates nuclear factor (NF)- $\mathrm{BB}$ and p38 MAP kinases via the ST2/IL-33 axis $(27,28)$. A recent mechanistic study (29) further elucidated that binding of IL-33 with ST2 reduces ST2 mobility to engage additional interaction with IL-1RAcP in the IL-1RAcP-ST2-IL-33 complex. Activation of the 
ST2/IL-33 axis in Th2 cells causes secretion of IL-4, IL-5, IL-13 (21, 28, 30), and IL-9 (31) that elicit the type 2 immune response (32). IL-33 signaling also promotes regulatory function of ST2 ${ }^{+}$Tregs in the colon and restrains inflammation in a murine acute colitis model (33) and GVHD (34). Depending on the cellular stimuli, sST2 can be secreted by endothelial cells, fibroblasts, epithelial cells, and type $1 \mathrm{CD} 4^{+}$and CD ${ }^{+}$ T cells (34). sST2 sequesters free IL-33 to attenuate the type 2 immune response, such as in allergic airway inflammation (35), and can contribute to disease progression when dysregulated.

GVHD is the major life-threatening complication in patients receiving allogeneic HCT. In HCT, damage to recipients' tissues, caused by preconditioning regimens, enhances the antigen-presenting cell-mediated proinflammatory response to donor T cells during transplantation, which contributes to GVHD development (36). At the early onset of GVHD, excess sST2 production dampens the type 2 immune response, disables its balanced control of type 1 immunity, and exacerbates disease progression $(12,34,37)$. We have recently shown that therapeutic blockade of excess sST2 can mitigate the immune response in murine GVHD models (34). In our study of mice with induced GVHD, we observed an initial major sST2 production locally from intestinal stromal and endothelial cells in the gastrointestinal (GI) tract followed by subsequent sST2 secretion from alloreactive T cells. Mice that were treated with an ST2-neutralizing antibody during HCT exhibited decreased plasma sST2 concomitant with increased plasma IL-33, decreased production of Th1 cells/cytotoxic T cells (Tc1) and Th17/ Tc17 cells, and increased ST2 expression on Th2 cells and Tregs during GVHD development (34). Although the ST2 antibody binds both ST2 and sST2, we reasoned that circulating ST2 antibody neutralizes the rise in local and systemic sST2 levels during the onset of GVHD to ameliorate GVHD progression. Displacement of sST2 from IL-33 by the ST2 antibody may lead to SST2 becoming prone to proteolytic degradation in the blood stream and decrease sST2 concentration over time. A precedent example is in the finding that association of IL-6 and its soluble receptor prolongs their half-lives in circulation (38).

The feasibility of using small molecules to inhibit cytokine receptors, such as ST2, to achieve therapeutic benefit is unknown because biologics are the traditionally used antagonists (39). Motivated by our findings in GVHD models using the ST2 antibody and the advantages of small-molecule therapies over antibodies, including easier administration especially by the oral route, superior tissue penetration, modifiable pharmacokinetic properties, higher uniformity between batches, and lower manufacturing costs, we combined high-throughput screening (HTS) and computational analyses to discover 3 chemical series of small-molecule ST2 inhibitors. After toxicity evaluation, we selected 3 candidate compounds for in vivo studies. We found that administration of 2 compounds led to reduced sST2 levels and amelioration of GVHD without compromising the graft-versus-leukemia (GVL) effect in mouse models. Because the ST2/ IL-33 axis plays important roles in the pathogeneses of other inflammatory diseases (40), therapeutics derived from these compounds may have applications beyond GVHD.

\section{Results}

Identifying small-molecule inhibitors of ST2. To discover ST2 inhibitors, we have developed a biochemical AlphaLISA that measures the binding between ST2-Fc (fragment, crystallizable) and IL-33. The AlphaLISA was then implemented and optimized in a pilot screening of 2,727 compounds with known biological activity. The robustness of the assay was determined by the $Z^{\prime}$ factor that corresponds to a combination of the signal-to-basal ratio (S/B) with the coefficient of variation (CV). For the pilot screening, we obtained an $\mathrm{S} / \mathrm{B}=11$ and $\mathrm{CV}=0.7$, giving a $Z^{\prime}$ factor of $0.95(n=2)$. One hit (sappanone $\mathrm{A}$ ) from the pilot screening was confirmed in secondary biochemical assays, but it was not further evaluated because of its cytotoxicity to human peripheral blood mononuclear cells (hPBMCs).

For the large-scale HTS experiment, we adopted the workflow outlined in Figure 1. In the primary screening, 77,701 compounds from 3 commercial suppliers (ChemBridge, ChemDiv, and Analyticon Discovery) were tested at a single dose $(17 \mu \mathrm{M})$ using the AlphaLISA. We obtained an average $Z^{\prime}$ factor of greater than 0.7 for all plates, and 1,823 compounds yielded inhibition of $30 \%$ or higher. To select a set of diverse and representative compounds for confirmatory screening, the 1,823 compounds were annotated by the Molecular ACCess System (MACCS) fingerprint (41) to enable structural comparison in the computational analysis. Tanimoto coefficients calculated based on the fingerprints of the compounds were used to quantify their structural differences, and a cutoff Tanimoto coefficient at 0.65 was set to cluster compounds into the same group. Each group was further inspected to remove frequent binders (found in either PubChem or an internal curated database) and compounds containing substructures alerted in the pan-assay interference compounds (PAINS) (42). Remaining compounds 


\section{Flowchart of Integrated High Throughput Screening and Computational Analysis to Discover ST2 Inhibitors}

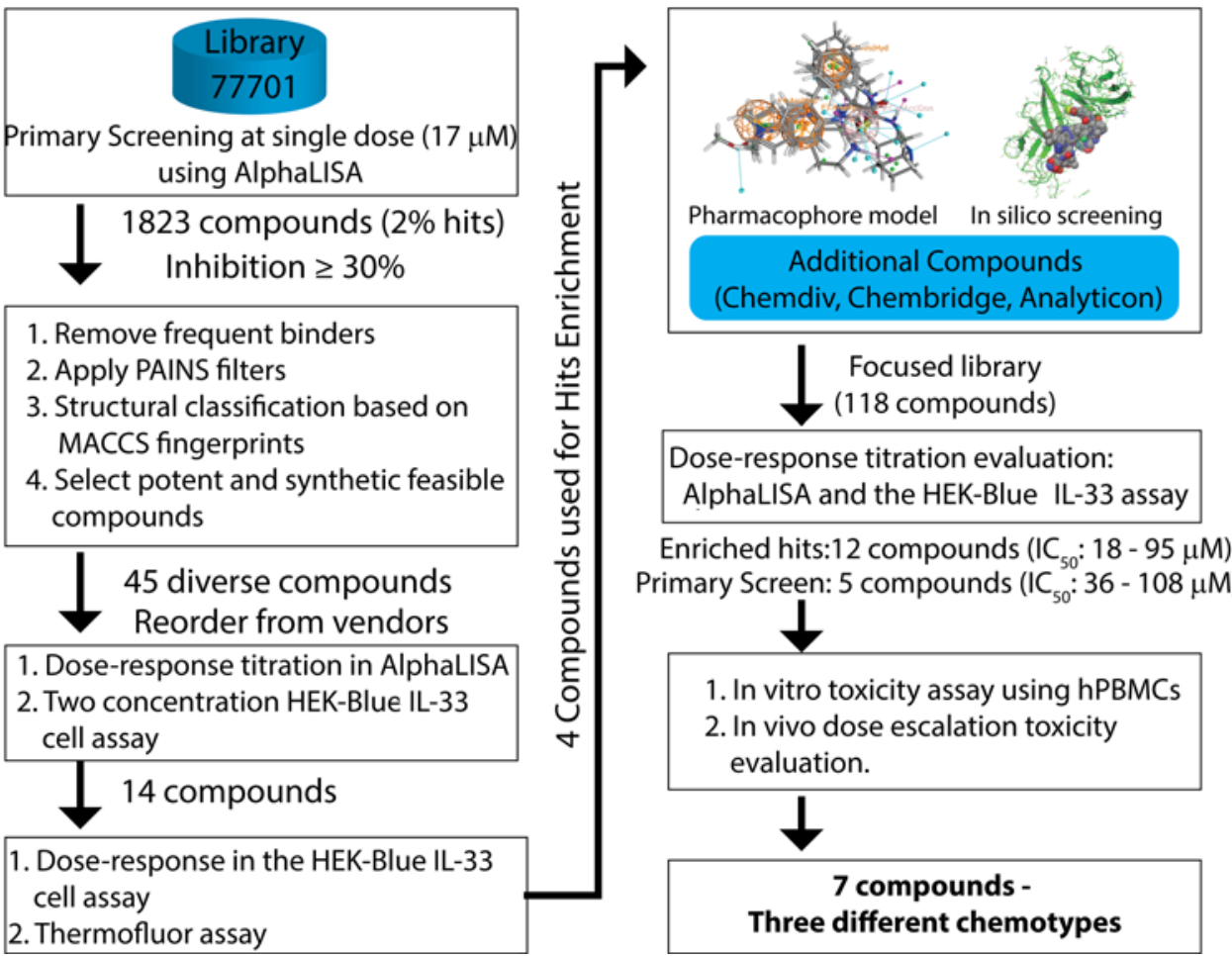

Figure 1. Flowchart of integrated high-throughput screening and computational analysis for discovery of ST2 inhibitors. PAINS, pan-assay interference compounds; MACCS, Molecular ACCess System; hPBMC, human peripheral blood mononuclear cell.

amenable to further chemical modifications were selected after consultation with medicinal chemists. These computer-based compound analyses led to the selection of 45 representative compounds for secondary confirmatory evaluation, and we found that 14 of the 45 compounds gave dose-dependent titrations. In addition to the AlphaLISA, we used another confirmatory Thermofluor assay to identify 4 compounds of the 14 that showed direct binding activities to 2 constructs of our recombinant ST2 proteins, as indicated by positive shifts in protein unfolding temperature (Figure 1).

To examine the specificity of the 14 compounds for ST2/IL-33 inhibition, we employed the human IL-33 sensor cells HEK-Blue IL-33 cells (InvivoGen). HEK-Blue IL-33 cells were generated by stable transfection of HEK293-derived cells with the human IL1RL1 gene to overexpress ST2 on the cell membrane. This modification was augmented by blockade of the tumor necrosis factor- $\alpha$ (TNF- $\alpha)$ receptor and IL-1R1 on the cell membrane to permit specific receptor response to IL-33 signaling. Activation of the NF-kB and AP-1 pathways transduced by the ligation of bioactive IL-33 with ST2 on the HEK-Blue IL-33 cell is monitored by the amount of the secreted embryonic alkaline phosphatase (SEAP) reporter proteins. In this experiment, we also included TNF- $\alpha$ ligand to confirm the inactivity of the TNF- $\alpha$ receptor pathway as a negative control. In the HEK-Blue IL-33 cell-based assay, 10 of the 14 compounds gave $40 \%$ or greater inhibition at $17 \mu \mathrm{M}$.

Based on the data of these assays, 4 hits (Supplemental Figure 1; supplemental material available online with this article; https://doi.org/10.1172/jci.insight.99208DS1) were selected as the basis for the subsequent ligand- and receptor-based computer screening in the hits enrichment study. In the ligand-based approach, we aligned the 3-D structures of the 4 hits to determine the common pharmacophore features and their distance relationship. A 4-feature pharmacophore model was then used as a filter to search commercial compound libraries. In the receptor-based screening, we performed a structure-based screening against the IL-33 binding site in ST2 (see Methods) using an indole surrogate library from Chem-X-Infinity. These analog searches led to selecting 118 additional candidates for activity evaluation. Among them, 12 
compounds exhibited dose-dependent titration, giving $\mathrm{IC}_{50}$ value ranges of $17.97-95.08 \mu \mathrm{M}$ in the AlphaLISA and 23.9-76.5 $\mu \mathrm{M}$ in the HEK-Blue assay. Inhibition activities of the extensive list of initial hits and their analogs have recently been published in a patent (WO 2017083242A1).

In vitro and in vivo toxicity evaluation of selected candidates. To select compounds suitable for in vivo evaluation, we first assessed the acute toxicity of 17 compounds (including 12 compounds identified from hits enrichment) in an in vitro assay using hPBMCs. After 10 hours of incubation at 3 concentrations (7.4-66 $\mu \mathrm{M}), 10$ compounds killed $10 \%$ or less of hPBMCs at $22.2 \mu \mathrm{M}$, and 6 of them killed less than $20 \%$ of $\mathrm{hPBMCs}$ at $66 \mu \mathrm{M}$ (Figure 2A), which is comparable to the control, citalopram (a serotonin transporter inhibitor and a false positive identified from the pilot screening). Structurally, the 6 less toxic compounds can be classified into 3 distinct chemotypes (I-III in Supplemental Figure 2), of which chemotype III compounds contain a quincoridine core derived from natural products. Despite their lower in vitro toxicity, we reasoned that the thione group of CB6141343 in chemotype I may be prone to oxidation during in vivo metabolism. CB6141343 was replaced by iST2-1 and CD4170-0776 discovered from analog searching. For compounds in chemotype II, we found that iST2-2 has improved solubility relative to CD3573-0071 and another analog of iST2-2, iST2-3, was also included for comparison. The $\mathrm{IC}_{50}$ values of iST2-1 determined by the AlphaLISA and the HEK-Blue assay were 56.14 and $54.62 \mu \mathrm{M}$, respectively, and for iST2-2, they were 122 and $25.93 \mu \mathrm{M}$, respectively (Figure 2B). Notably, iST2-3 was 2-fold more potent than iST2-2 in both assays (Figure 2A). iST2-4 from chemotype III had $\mathrm{IC}_{50}$ values of $17.57 \mu \mathrm{M}$ (AlphaLISA) and $25.92 \mu \mathrm{M}$ (HEK-Blue) (Figure 2B). Ultimately, 7 compounds from 3 chemotypes (Supplemental Figure 2) were chosen for the 16-day in vivo dose escalation toxicity study in which mice were treated with each compound at $5,10,20$, or $40 \mathrm{mg} / \mathrm{kg}$ on days $1,4,9$, and 12 . Only CD4170-0776 showed toxicity in mice starting at $10 \mathrm{mg} / \mathrm{kg}$ (Figure 2C) and was subsequently eliminated. For the remaining 6 compounds, only iST2-3 showed toxicity to mice at a higher dose (one mouse died after treatment with $40 \mathrm{mg} / \mathrm{kg}$ iST2-3 on day 12). Using the in vitro microsomal stability assay, we also found that iST2-1, 2, 3, and 4 can undergo quick first-passage clearance in vivo in mice (Supplemental Table 3). We anticipated that they would have acceptable toxicity and low accumulation in mice over extended periods of time.

Determination of the functional groups that contribute to the inhibitory activity of iST2-1. Among the 3 representative compounds in Figure 2B, the commercially acquired iST2-1 and iST2-2 are racemic mixtures that contain 2 stereospecific isomers (Figure 3A). To identify important functional groups of our ST2 inhibitors that produced their activities, we studied iST2-1 because of its higher potency and synthetic feasibility. We first synthesized racemic iST2-1, $(S)$-iST2-1, $(R)$-iST2-1, and analogs of racemic iST2-1 shown in Figure 3A. In our AlphaLISA, we obtained an $\mathrm{IC}_{50}$ value of $47.7 \pm 5 \mu \mathrm{M}(n=5)$ for racemic iST2-1, which is close to $56.14 \mu \mathrm{M}$ of the commercially procured iST2-1. Based on the same assay, $(R)$-iST2-1 and $(S)$-iST2-1 had $\mathrm{IC}_{50}$ values of $43.0 \pm 15.1$ and $42.0 \pm 11.5 \mu \mathrm{M}(n=2)$, respectively, which are comparable to that of the racemic iST2-1 within curve fitting errors (Figure 3B). Using racemic iST2-1 as a reference, we found that replacement of the nitroso group in iST2-1 with a smaller fluorine atom (iST2-1-1F), a carboxylic acid (iST2-2), an amide group (iST2-3), or a cyano group (iST2-4) all reduce the inhibition constant by 2- to 5-fold. Removal of the methoxy group of iST2-1 gave iST2-5, which had a higher $\mathrm{IC}_{50}$ value at $97.56 \pm 28.2 \mu \mathrm{M}(n=2)$, too. The structure-activity relationship of select iST2-1 analogs showed that the nitroso and methoxy groups in iST2-1 contribute to its activity and warrant further exploration in inhibitor optimization.

Studies of the binding interaction between iST2-1 and ST2. Currently, no cocrystal structures of ST2 with iST2-1 or any small molecule are available. We and other groups $(43,44)$ have previously shown that the D3 domain in the ST2 ectodomain (ST2 ${ }^{\mathrm{ECD}-\mathrm{D} 3}$ ), anchored by a preceding linker region to the $\mathrm{D} 2$ domain, exhibits high mobility in apo-ST2 (the ligand-free ST2). Our computational studies of IL-1R1 and ST2 also indicated that the ectodomain of cytokine receptors may possess conformations that can be modulated by allosteric inhibitors $(44,45)$. To investigate if iST2-1 imposed allosteric inhibition in solution, we conducted small-angle X-ray scattering (SAXS) studies. In Figure 3C, we show that the SAXS profile of ST2 $2^{\mathrm{ECD}} / \mathrm{iST} 2-1$ is close to that of apo-ST2 ${ }^{\mathrm{ECD}}$. The Kratky plot in Figure 3C indicated that ST2 ${ }^{\mathrm{ECD}} / \mathrm{iST} 2-1$ retains the asymmetric globular shape of apo-ST2 ${ }^{\mathrm{ECD}}$ except a slight increase at $0.05<q<0.15$ attributable to the addition of iST2-1 to apo-ST2 $2^{\mathrm{ECD}}$. Although the calculated pair-wise distance distribution, $P(r)$, of ST2 $2^{\mathrm{ECD}} / \mathrm{iST} 2-1$ differs from that of apo-ST2 $2^{\mathrm{ECD}}$, comparison of their reconstructed ab initio shape functions indicated that iST2-1 binding made minor modifications to the overall shape of apo-ST2 ${ }^{\mathrm{ECD}}$ (Figure 3D). Analysis of the SAXS profiles gave radius of gyration $\left(R_{g}\right)$ values of 31.38 and $31.26 \AA$ for apo-ST2 and ST2 ${ }^{\mathrm{ECD}} / \mathrm{iST} 2-1$ (Supplemental Table 1), suggesting similar conformational states between them. The same analysis also gave lower esti- 

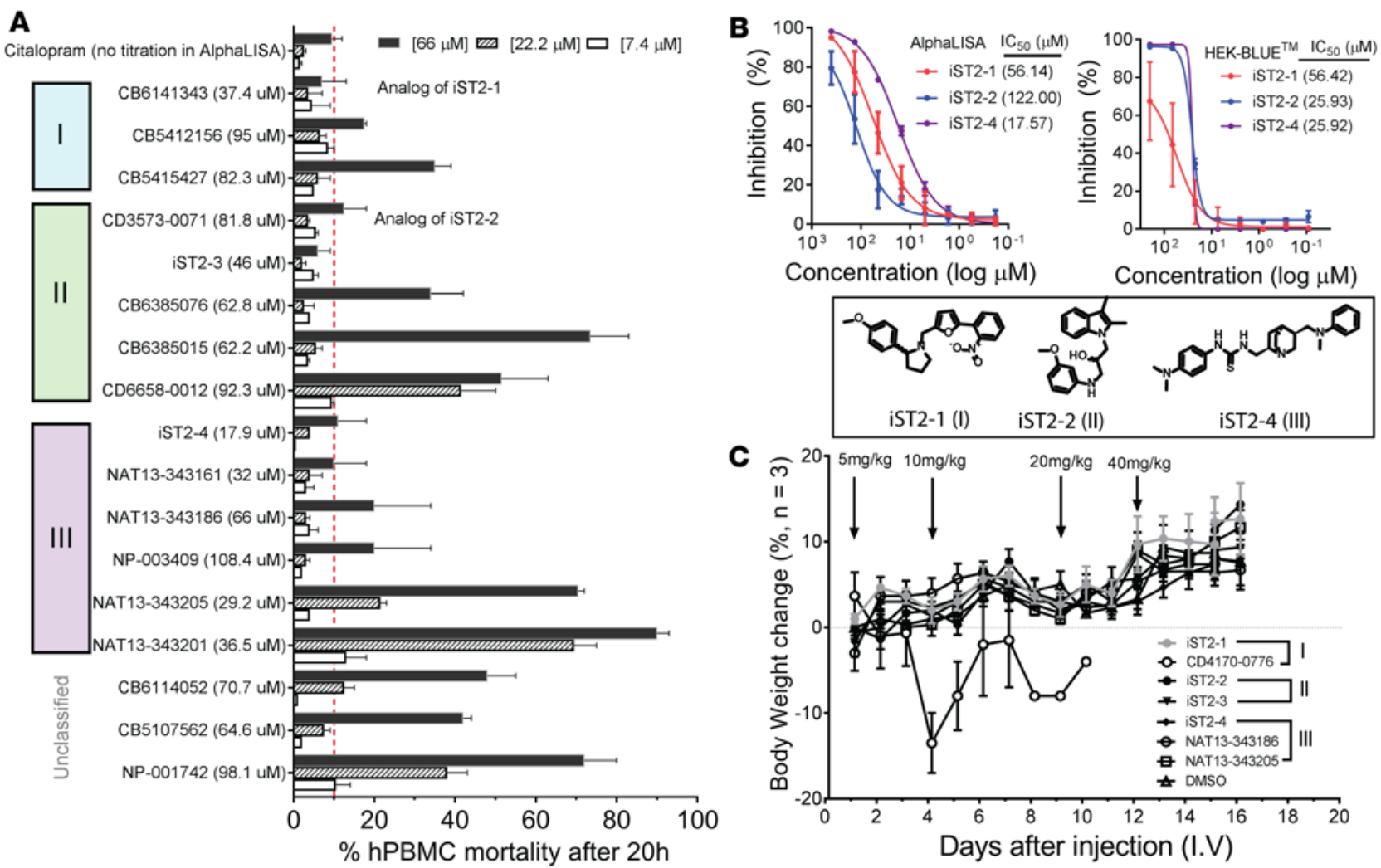

Figure 2. In vitro and in vivo toxicity evaluation of selected candidates. (A) Human PBMC death induced by compounds at 3 concentrations after incubation for 20 hours (duplicate per compound at each concentration). $\mathrm{IC}_{50}$ values determined by the AlphaLISA are shown in parentheses. Compounds belonging to chemotypes I, II, and III and unclassified are grouped together. CB6114052, CB5107562, and NAT13-343201 are 3 initial hits. The average IC ${ }_{50}$ values of 17 compounds at $63.7 \mu \mathrm{M}$ were used as references for setting the maximum concentration at $66 \mu \mathrm{M}$. (B) Titration curves for iST2-1, iST2-2, and iST2-4 measured with the AlphaLISA and the HEK-Blue IL-33 assay. (C) In vivo dose-escalation toxicity evaluation of 7 selected compounds in healthy mice. Data represent mean \pm SEM ( $n=3$ per compound). hPBMC, human peripheral blood mononuclear cell.

mated molecular weights (MWs) of 42.4 and $42.8 \mathrm{kDa}$ for apo-ST2 and ST2 2 ECD $/$ iST2-1 than those (between 43.9 to $44.8 \mathrm{kDa}$ ) determined by liquid chromatography-mass spectroscopy (LC-MS) analysis for apo-ST2 ${ }^{\mathrm{ECD}}$ (Supplemental Figure 3). Taken together, the SAXS studies suggest that binding of iST2-1 to ST2 ${ }^{\mathrm{ECD}}$ causes small changes to the conformational flexibility of ST2 $(46,47)$ and may not restrict the mobility of ST2 ${ }^{\mathrm{ECD}-\mathrm{D} 3}$.

To determine potential $(R)$ - and $(S)$-iST2-1 binding sites in ST2 ${ }^{\mathrm{ECD}}$, we employed unbiased molecular dynamics (MD) simulations. The binding site detection is enhanced by a modified cosolvent MD simulation method (cMD) (48) that embeds ST2 $2^{\mathrm{ECD}}$ in an environment that is high in $(R)$-iST2-1 or $(S)$-iST2-1 concentration. The high inhibitor concentration increases the encounter probability between the inhibitor and different binding sites in $\mathrm{ST} 2^{\mathrm{ECD}}$ simultaneously during the simulations. This implementation overcomes the need to perform lengthy and multiple MD simulations by placing a single inhibitor in random positions relative to the protein in simulations (49). After the encounter between inhibitors and putative binding sites in $\mathrm{ST} 2^{\mathrm{ECD}}$, the stronger $\mathrm{ST} 2^{\mathrm{ECD}}$-inhibitor interaction will theoretically retain the inhibitor at the sites for a longer time compared with transient association. To detect recurring (or consensus) binding sites, 2 different simulation conditions were performed (see Methods).

Because the SAXS data indicated that iST2-1 may not modulate the conformation of ST2 ${ }^{\mathrm{ECD}-\mathrm{D} 3}$, we focused our analyses of iST2-1-binding sites on the D1 and D2 domains of ST2 (ST2 ${ }^{\text {ECD-D1D2}}$ ). ST2 ${ }^{\text {ECD-D1D2 }}$ accounts for $83 \%$ of the binding free energy between ST2 and IL-33 and contains potential small-moleculebinding sites (44). We found that iST2-1 resides persistently in multiple locations on ST2 ${ }^{\mathrm{ECD}}$ during the 32 ns of simulation time. Consensus sites include S1r, and S2r for $(R)$-iST2-1, and S1s for (S)-iST2-1 shown in Figure 3E. Although S1r, located between the D2 and D3 domains of ST2, coincides with small-moleculebinding hotspots characterized in our previous study (44), 2 other consensus sites are not at the interaction interface between ST2 and IL-33. At the ST2/IL-33 interaction interface, 3 non-consensus binding sites 
A

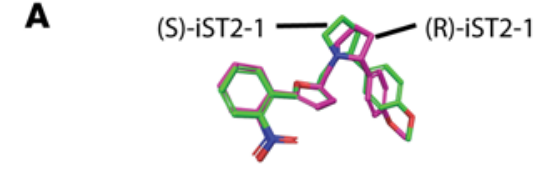<smiles>COc1ccc(C(CO)c2ccc(-c3ccccc3OC)cc2)cc1</smiles>

iST2-1 $(47.7 \pm 5.0 \mu \mathrm{M})$

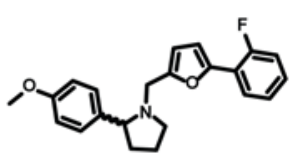

iST2-1F $(115.2 \pm 36.9 \mu \mathrm{M})$

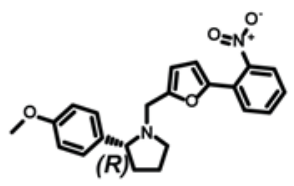

(R)-iST2-1 (43.0 $\pm 15.1 \mu \mathrm{M})$

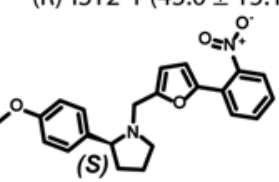

(S)-iST2-1 (42.0 $\pm 11.5 \mu \mathrm{M})$

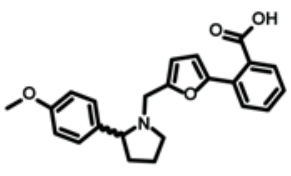

iST2-1a $(219.7 \pm 14.8 \mu \mathrm{M})$

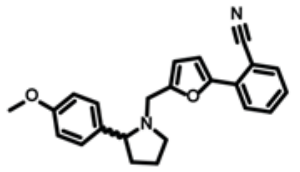

iST2-1c (116.3 $\pm 9.5 \mu \mathrm{M})$

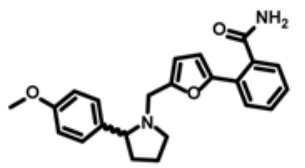

iST2-1b $(167.2 \pm 45.7 \mu \mathrm{M})$

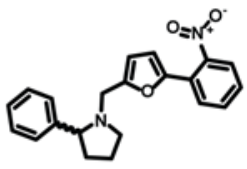

iST2-1d (97.56 $\pm 28.2 \mu \mathrm{M})$

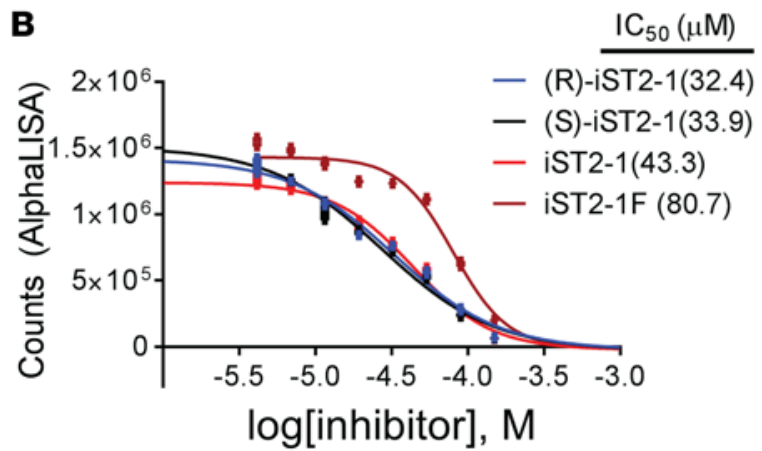

C
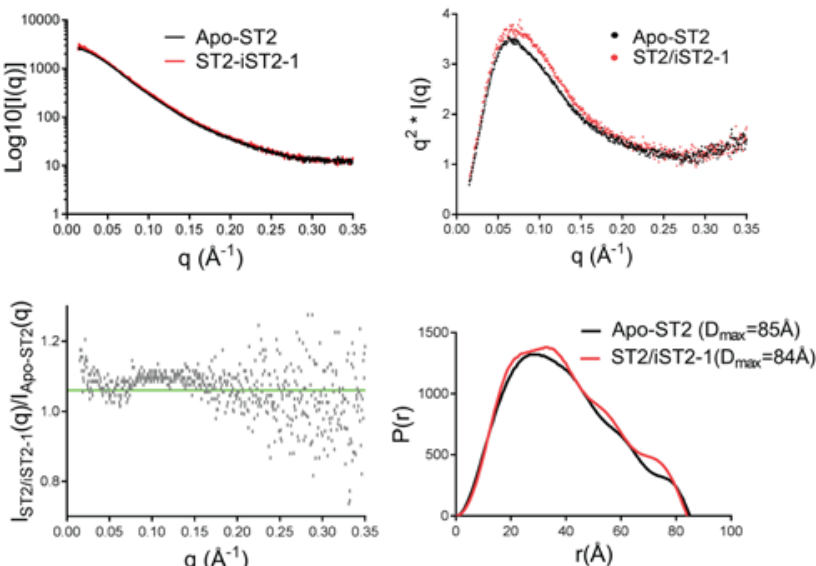

D
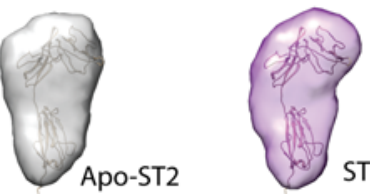

$\mathrm{ST} 2 / \mathrm{iST} 2-1$

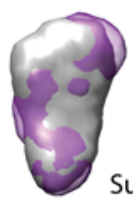

Superposition

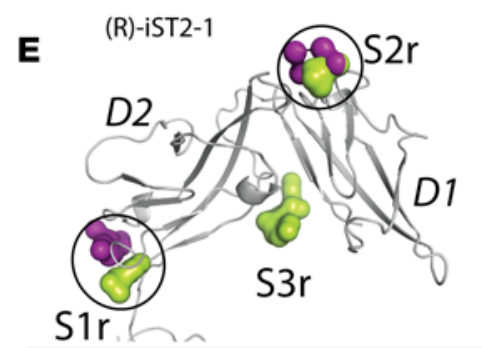

(S)-iST2-1

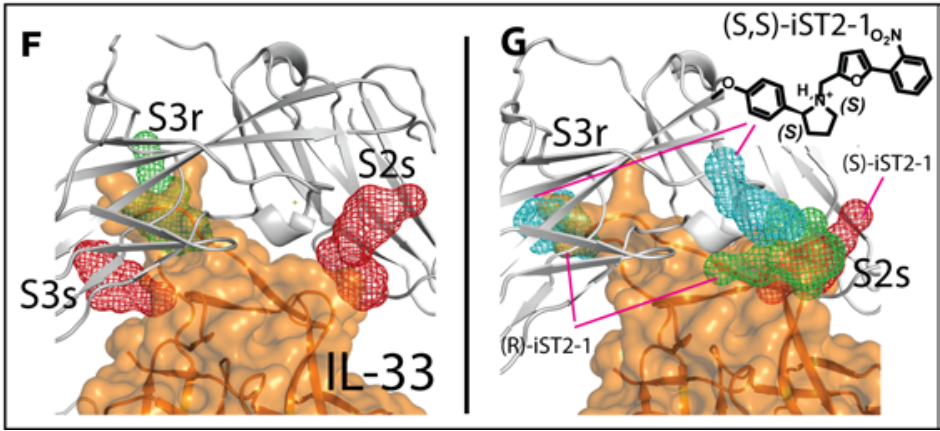

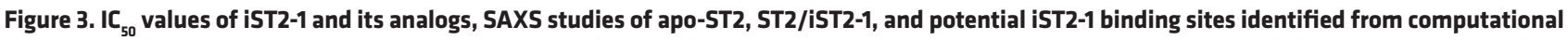
simulations. (A) Chemical structures of racemic iST2-1, (R)-iST2-1, (S)-iST2-1, analogs of iST2-1, and structural alignment of (R)-iST2-1 (purple) with (S)-iST2-1 (green). $\mathrm{IC}_{50}$ values of the inhibitors are shown in parentheses. (B) Inhibition curves and $\mathrm{IC}_{50}$ values (in parentheses) of 4 representative compounds. Each data point is an average of triplicate measurements \pm SD. (C) SAXS profiles, the residual plot between the SAXS profiles of apo-ST2 and ST2/iST2-1, comparison of the Kratky plot based on the SAXS profiles of apo-ST2 and ST2/iST2-1, the pair-wise distance distribution [P(r)] of apo-ST2 and ST2/iST2-1 calculated from the SAXS profiles. $D_{\max }$ values are shown in parentheses. (D) Ab initio shape reconstruction of apo-ST2 (gray) and ST2/iST2-1 (purple). (E) Mapping of the (R)iST2-1 and (S)-iST2-1 binding sites (surface envelop) in ST2 based on 32-ns MD simulations. Maps detected from 13- and 15-Å octahedron boxes are colored in purple and green, respectively. Consensus binding sites (S1r, S2r and S1s) are circled. The D1 and D2 domains of ST2 are labeled. (F) Binding sites of (R)-iST2-1 (green mesh) and (S)-iST2-1 (red mesh) that block interaction between ST2 and IL-33 (orange) are highlighted. (G) Binding sites of (R)-, (S)-, (S,S)-iST2-1 in the glycosylated ST2 from the MD simulations. (S,S)-iST2-1 is (S)-iST2-1 in which the proton on the pyrrolidine group is in the $S$ form and its mapped site is shown in cyan mesh. SAXS, small-angle X-ray scattering.

of (R)-iST2-1 (S3r) and (S)-iST2-1 (S2s, S3s) were also identified that may directly inhibit the association between ST2 and IL-33 (Figure 3, E and F). To examine if the force-field parameters and glycosylation of $\mathrm{ST}^{\mathrm{ECD}}$ may affect the $\mathrm{ST} 2^{\mathrm{ECD}}$ conformation to exclude these detected binding locations of iST2-1, we performed additional simulations using the monoglycosylated ST2 ${ }^{\mathrm{ECD}}$ resolved in the ST2/IL-33 structure with the most recent force-field parameters and studied 2 additional chiral structures of iST2-1 related to 
the protonation of the pyrroline group in iST2-1. We confirmed that $(R)$-iST2-1, $(S)$-iST2-1, and $(S, S)$ iST2-1 bind preferentially to S3r and S2s sites (Figure 3G). Because S3r and S2s are in close proximity, whether $(R)$-iST2-1 and (S)-iST2-1 compete for a same binding site remains to be determined.

$S T 2$ inhibitors decrease IFN- $\gamma$-producing $T$ cell populations in the in vitro mixed lymphocyte reaction assay. To examine the utility of our ST2 inhibitors in GVHD, we first evaluated iST2-1, iST2-2, and iST2-4 (Figure 2B), representing 3 different chemotypes, in the in vitro human mixed lymphocyte reaction (MLR) assay. In the MLR, purified $\mathrm{CD}^{+}$and $\mathrm{CD} 8^{+} \mathrm{T}$ cells from a healthy donor (responder) are mixed with irradiated $\mathrm{T}$ cell-depleted PBMCs from a second healthy HLA-mismatched donor (stimulator) to model alloreaction of $\mathrm{T}$ cells responding to alloantigens in vitro $(50,51)$. Similar to GVHD, the T cells' alloreactivity in the MLR can induce sST2 secretion to dampen Th2 activity and shift T cell polarization to Th1. In the DMSO control, we observed continued replication/proliferation of $\mathrm{CD}^{+} \mathrm{IFN}_{-} \gamma^{+}(2.25 \%)$ and $\mathrm{CD} 8^{+} \mathrm{IFN}-\gamma^{+}(17.9 \%) \mathrm{T}$ cells after 7 days in the MLR. In the MLR incubated with iST2-1 from 1 to $10 \mu \mathrm{M}$, dose-dependent reduction of $\mathrm{CD} 4+\operatorname{IFN}-\gamma^{+}(1.51 \% \rightarrow 0.32 \%)$ and $\mathrm{CD}^{+} \mathrm{IFN}^{-} \gamma^{+}(15.6 \% \rightarrow 1.57 \%) \mathrm{T}$ cell populations were observed (Supplemental Figure 4A). Although iST2-4 was more potent than iST2-1 in biochemical and cell-based assays, it was less effective at reducing the IFN- $\gamma^{+} \mathrm{T}$ cell population. Relative to the DMSO control, the least potent compound, iST2-2, only reduced the CD $4^{+} \mathrm{IFN}-\gamma^{+} \mathrm{T}$ cell population by $39 \%$ at $10 \mu \mathrm{M}$, whereas $86 \%$ and $81 \%$ reductions in the $\mathrm{CD}^{+} \mathrm{IFN}-\gamma^{+} \mathrm{T}$ cell population were observed in the iST2-1- and iST2-4-treated groups, respectively. Treatment with any of the $3 \mathrm{ST} 2$ inhibitors maintained the beneficial FoxP $3^{+} \mathrm{CD} 4^{+}$Treg population compared with DMSO control (Supplemental Figure 4B). In vitro MLR assay data demonstrated that our ST2 inhibitors - by potentially blocking the sequestration of IL-33 by sST2 - attenuated Th1/Tc1 cell proliferation compared with DMSO control while maintaining the Treg population.

$S T 2$ inhibitors reduce plasma sST2 levels, alleviate GVHD, and improve survival in vivo. After assessing the in vivo toxicity results, the MLR data, and the feasibility of procuring the compounds in a timely manner, 3 racemic compounds - iST2-1, iST2-2, and iST2-3 - were selected for testing in 2 experimental GVHD disease models. In the first xenogeneic model, $10^{6} \mathrm{~T}$ cells extracted from hPBMCs (hu-T cells) were transplanted into NOD-scid IL2Rgamma ${ }^{\text {null }}$ (NSG) mice preconditioned with $300 \mathrm{cGy}$ total body irradiation (TBI) on day -1 . In the second minor HLA-mismatched model, $2 \times 10^{6} \mathrm{~T}$ cells and $5 \times 10^{6}$ bone marrow (BM) cells from C57BL/6 (B6) mice were transplanted into C3H.SW mice that received 1,100 cGy TBI on day -1 . Mice in both models received twice daily intraperitoneal (i.p.) injections of ST2 inhibitors (iST2-1, $41.4 \mathrm{mg} / \mathrm{kg} / \mathrm{day}$; iST2-2, $18 \mathrm{mg} / \mathrm{kg} /$ day; and iST2-4, $20.5 \mathrm{mg} / \mathrm{kg} /$ day) or DMSO as control for 21 days starting from day -1 to day 20 (Figure $4 \mathrm{~A}$ ). In the hu-T cells $\rightarrow$ NSG model, we examined the effects of ST2 inhibitors on human T cell differentiation, whereas in the $\mathrm{B} 6 \rightarrow \mathrm{C} 3 \mathrm{H}$.SW model, the impact of ST2 inhibitors on mouse $\mathrm{T}$ cell polarization was measured.

Ex vivo analysis showed that iST2 inhibitor treatment did not change the number of infiltrating $\mathrm{T}$ cells in the gut, which is the main direct GVHD target organ, compared with control in the $\mathrm{B} 6 \rightarrow \mathrm{C} 3 \mathrm{H} . \mathrm{SW}$ model (Figure 4B). In both models treated with iST2-1 and iST2-2, we observed a reduction in CD4 $4^{+} \mathrm{IFN}-\gamma^{+} \mathrm{T}$ cells and an increase in $\mathrm{FoxP}^{+} \mathrm{CD}^{+}$Tregs at day 14 after HCT (Figure $4 \mathrm{C}$ and Supplemental Figure 5). In the B6 $\rightarrow \mathrm{C} 3 \mathrm{H}$. SW model, the iST2-1 and iST2-2 treatment groups also showed decreases in both the Th1 (CD4+T-bet $\left.{ }^{+}\right)$and Th17 (CD4 $\left.{ }^{+} \mathrm{ROR} \gamma \mathrm{t}^{+}\right)$cell populations. On day 21, the iST2-1 treatment group showed effective control of the $\mathrm{CD} 4{ }^{+} \mathrm{IFN}-\gamma^{+}$T cell population, at only $2.5 \%$ in the hu-T cells $\rightarrow$ NSG model and $40 \%$ in the B6C3H.SW model compared with CD4 $4^{+} \mathrm{IFN}-\gamma^{+}$T cell populations of $20 \%$ and $70 \%$ with the DMSO control, respectively (Figure 4, D and E, and Supplemental Figure 6). Increases in the FoxP3 ${ }^{+} \mathrm{CD} 4^{+}$Treg population (Supplemental Figure 7) were also observed in mice treated with iST2-1 compared with the DMSO control in both the xenogeneic and minor HLA-mismatched models ( $10 \%$ versus $<1 \%$ in the hu-T cells $\rightarrow$ NSG model and $10 \%$ versus $2 \%$ in the $\mathrm{B} 6 \rightarrow \mathrm{C} 3 \mathrm{H}$.SW model). A 2-fold increase in $\mathrm{CD}^{+} \mathrm{IL}-4$-secreting cells was found in the iST2-1 treatment group compared with the DMSO control (Supplemental Figure 7). Although CD8 ${ }^{+}$T cells can drive the GVHD progression in the $\mathrm{B} 6 \rightarrow \mathrm{C} 3 \mathrm{H}$.SW model, we found that $\mathrm{CD} 8^{+} \mathrm{T}$ cells were present at low frequency in the gut and did not seem to drive the pathogenicity of SST2 in the gut. $\mathrm{CD}^{+} \mathrm{T}$ cells detected in the gut showed no significant differences between the ST2-inhibitor-treated and control groups on day 21 (Supplemental Figure 8). Consistent with the in vitro MLR experiment, in vivo iST2-2 treatment was less effective at limiting CD4 $4^{+} \mathrm{IFN}-\gamma^{+} \mathrm{T}$ cell proliferation and increasing the FoxP3 ${ }^{+} \mathrm{CD} 4^{+}$Treg population over time.

Two important biomarkers (plasma levels of sST2 and IFN- $\gamma$ ) indicative of GVHD progression were monitored in this study. In the hu-T cells $\rightarrow$ NSG model, we measured human sST2 and IFN- $\gamma$ levels to determine the response of human $\mathrm{T}$ cells. In both models, increases in both biomarkers were found at day 7 or later (Figure 5A). Differential control of both biomarker levels by ST2 inhibitors coincided with the 
A
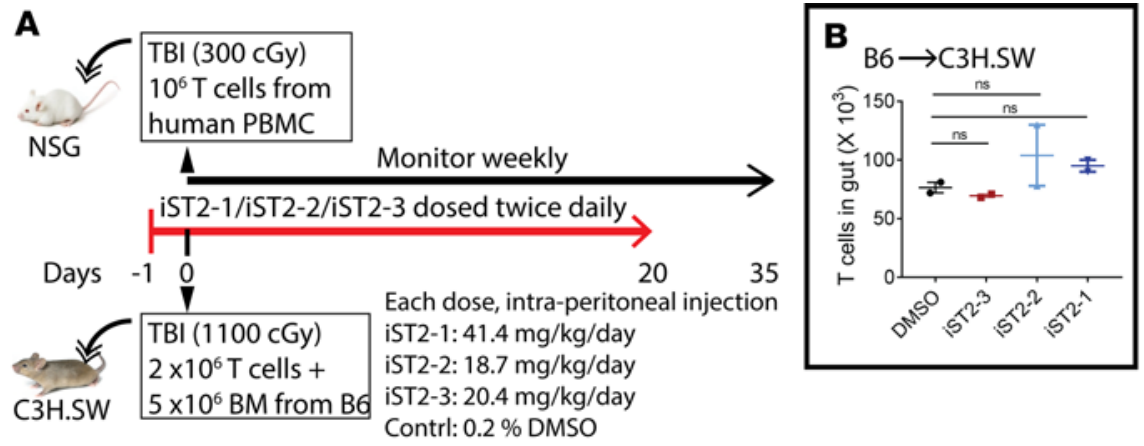

C day 14 (hu-T cells $\rightarrow$ NSG)

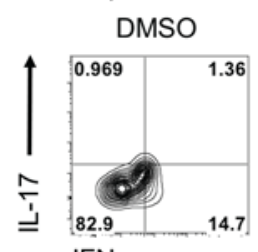

isT2-3

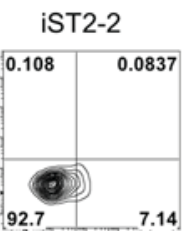

IFN $\gamma$
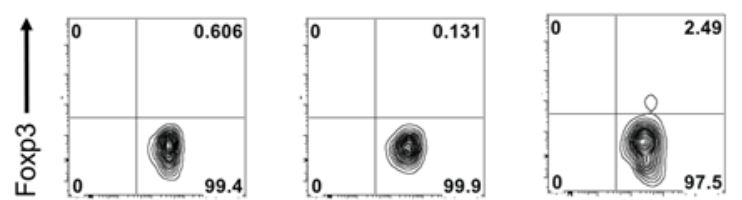

CD4

D day 21 (hu-T cells $\rightarrow$ NSG)
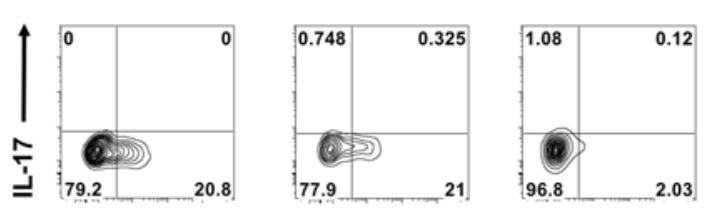

IFN $\gamma$
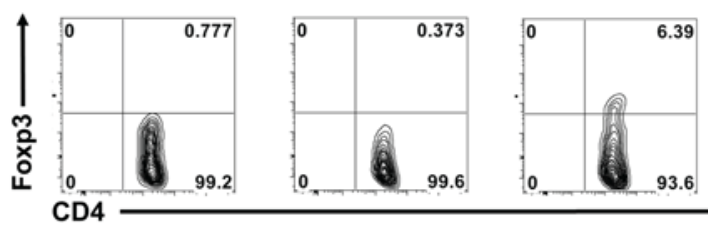

E day $21(\mathrm{~B} 6 \rightarrow$ C3H.SW $)$
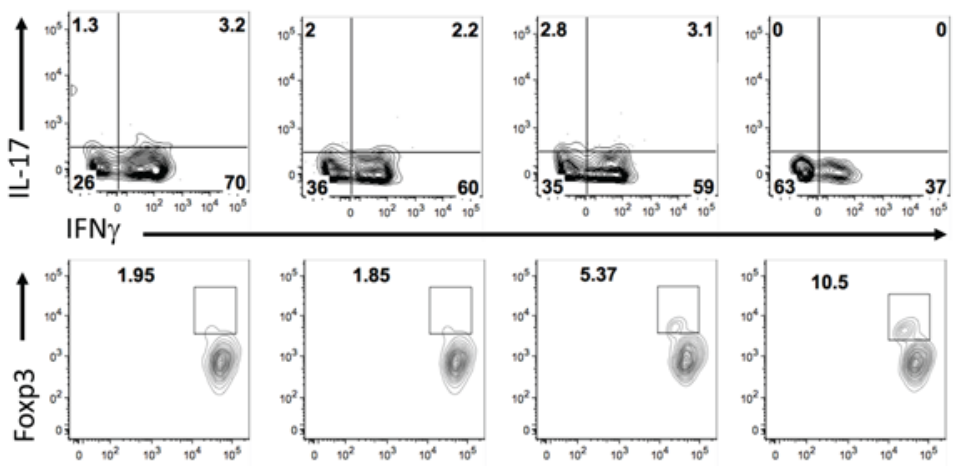

CD4
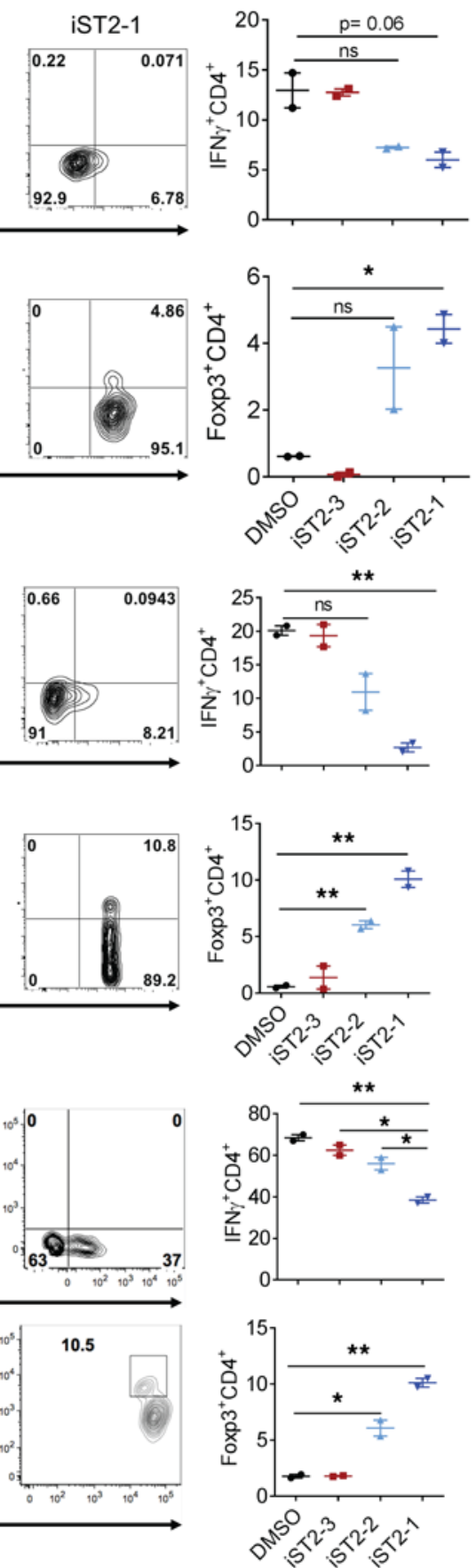

Figure 4. Dosages and treatment schedule for ST2 inhibitors and ex vivo analysis in the GVHD mouse models. (A) The dosages were calculated using the body weight of each mouse at $20 \mathrm{mg}$ and correspond to twice the $\mathrm{IC}_{50}$ values of the inhibitors determined by the HEK-Blue IL-33 assay. (B) Number of T cells infiltrating the gut in the B6C3H.SW GVHD model at day 14 . Flow cytometric analysis of intestinal $\mathrm{CD} 4^{+} \mathrm{IFN}-\gamma^{+}$and FoxP3 ${ }^{+} \mathrm{CD} 4^{+} \mathrm{T}$ cell populations on day 14 in the (C) hu-T cells $\rightarrow$ NSG model and day 21 in the (D) hu-T cells $\rightarrow$ NSG, (E) B6 $\rightarrow$ C3H.SW GVHD models treated with iST2-1, iST2-2, or iST2-3. $n=2$ per group at day 14 and 21 respectively, and for each model. Data represent mean $\pm \operatorname{SEM}(n=2)$. TBI, total body irradiation; hPBMC, human peripheral blood mononuclear cell. ${ }^{*} P<0.05,{ }^{* *} P$ $<0.01$ by unpaired $t$ test. 
bifurcation of GVHD scores starting on week 2 for the hu-T cells $\rightarrow$ NSG model and week 1 for the B6 $\rightarrow$ C3H.SW model (Figure 5, A and B). The results in Figure 5A show that iST2-1 treatment effectively prevented the escalation of sST2 levels in both models. Low levels of sST2 were maintained up to day 28 in the hu-T cells $\rightarrow$ NSG model and day 21 in the B6 $\rightarrow$ C3H.SW model. Plasma IFN- $\gamma$ levels increased on day 14 in the hu-T cells $\rightarrow$ NSG model and day 7 in the B6 $\rightarrow$ C3H.SW model. The iST2-1 treatment group showed more than 50\% lower plasma IFN- $\gamma$ levels than the DMSO control or the iST2-3 treatment group. In comparison, the reductions in plasma sST2 and IFN- $\gamma$ levels were less in mice treated with iST2-2.

Ex vivo cellular and plasma biomarker analyses showed the T cell population and GVHD-related biomarker changes affected by treatment with our ST2 inhibitors in 2 in vivo experimental models. In Figure 5B, we demonstrate that the most efficacious ST2 inhibitor, iST2-1, reduced the GVHD score to 3.25 in the hu-T cells $\rightarrow$ NSG model and to 4 in the $\mathrm{B} 6 \rightarrow \mathrm{C} 3 \mathrm{H} . \mathrm{SW}$ model accompanied by improved survival rates of $50 \%$ and $67 \%$, respectively, on day 35 . The less potent iST2-2 also reduced GVHD scores and improved survival rates to $25 \%$ and $50 \%$. The adverse outcome of iST2-3 treatment may be attributed to its increased toxicity to the disease-burdened mice, as suggested by the toxicity to healthy mice at a higher dose (Figure 2C). Taken together, our results showed that administration of iST2-1 or iST2-2 reduced the plasma sST2 levels, decreased GVHD scores, and improved survival in 2 GVHD models, and their in vivo efficacies correlated with decrease in the level of sST2.

iST2-1 ameliorates GVHD burden and maintains GVL effect in the murine MLL-AF9 leukemia model. The beneficial T cell alloreactivity is used to induce GVL activity to eliminate residual leukemia in HCT. To investigate whether treatment with iST2-1 ameliorated GVHD without diminishing the GVL effect, we studied the effects of iST2-1 treatment in the $\mathrm{B} 6 \rightarrow \mathrm{C} 3 \mathrm{H}$.SW model injected with syngeneic MLL-AF9 leukemia cells. iST2-1 was administered i.p. twice daily to the C3H.SW mice from day -1 to +20 after HCT. In this model, mice will succumb to either GVHD manifestations or leukemia without treatment. We found that animals treated with DMSO died of GVHD starting on week 3 and subsequently of leukemia for a $20 \%$ overall survival at the end of the study on day 73 (Figure 6). In the treatment group, mice receiving iST2-1 survived past day 70 and were GVHD/leukemia-free. Of note, this survival benefit was higher than expected, with the sole GVHD protection suggesting a potential increase in antitumor activity with iST2-1 treatment, similar to what we have observed with ST2-neutralizing antibody treatment (34). Thus, our data showed that amelioration of GVHD by iST2-1 treatment did not compromise and may have even increased the GVL benefit of HCT.

\section{Discussion}

The curative potential of allogeneic HCT for hematological malignancies and disorders has been limited by the frequent development of GVHD detected upon clinical presentation. Proteomics-based biomarker discovery in GVHD $(12,17,52-55)$ has now provided a means to stratify high-risk patients to receive aggressive disease management. Despite the progress made in prognosis, high-grade and steroid-refractory GVHD $(56,57)$ remains intractable. Biomarker studies have identified sST2 as the most significant prognostic biomarker in GVHD. sST2 produced by intestinal stromal cells, epithelial cells, and Tc/Th17 cells (34) can antagonize and suppress Th2 cell and Treg activation, leading to an amplified proinflammatory phenotype in GVHD development. Our previous study (34) indicated that reducing the levels of sST2 via ST2 antibody treatment can manage GVHD in mice. Small-molecule reagents capable of blocking the ST2-IL-33 interaction may produce similar therapeutic benefits to mitigate GVHD development and can be tailored to therapies for diseases using sST2 as a biomarker.

In this work, we have integrated computational analyses with HTS to discover 3 classes of smallmolecule ST2 inhibitors that block binding between ST2 and IL-33. In vitro and in vivo toxicity evaluations provided guidance of the selection of compounds exhibiting low or no toxicity for testing in followup disease model studies. The effects of iST2-1, -2, and -4 from 3 chemical classes on alloreactivity in the MLR assay further confirmed their effectiveness for treating alloreactive $\mathrm{T}$ cell activity in vitro. The efficacies of our ST2 inhibitors observed in 2 experimental models of GVHD demonstrated that they reduce the levels of prognostic biomarkers and Th1 cell populations. Although our biochemical assays were not designed to test the ability of our compounds to inhibit mouse ST2 ${ }^{\mathrm{ECD}}$ and IL-33, we hypothesized that our ST2 inhibitors target mouse ST2 because mouse and human ST2 ${ }^{\mathrm{ECD}}$ share $68 \%$ sequence identity and human ST2 $2^{\mathrm{ECD}}$ binds to human and mouse IL-33 (58). This is supported by the evidence that the ST2 inhibitors produced similar effects to reduce the same prognostic biomarkers in the human $\mathrm{T}$ cells to NSG mouse model and the $\mathrm{B} 6 \rightarrow \mathrm{C} 3 \mathrm{H}$.SW mouse GVHD model - where no human proteins are 
A
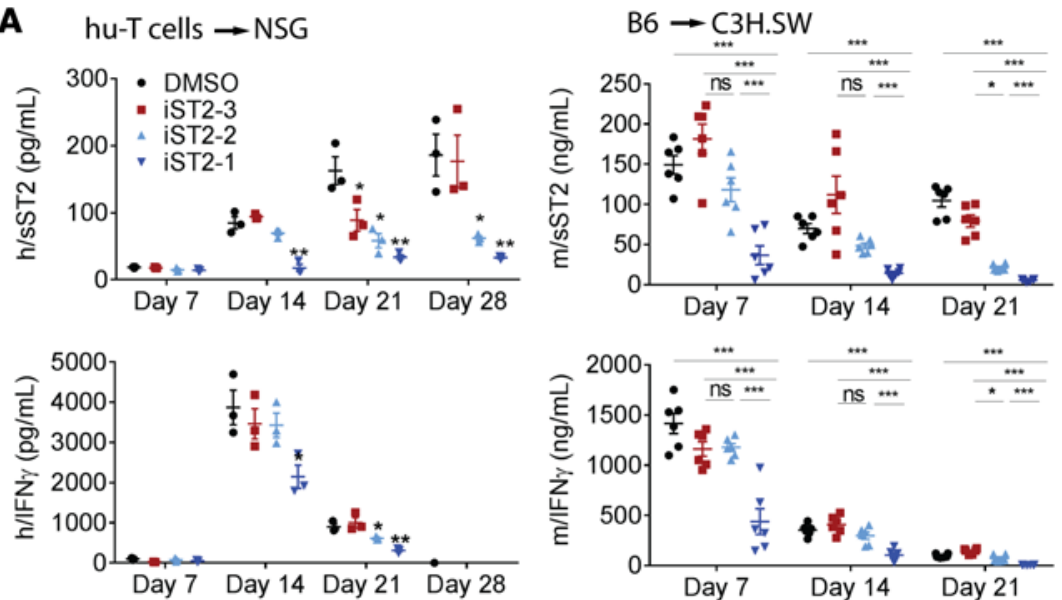

B
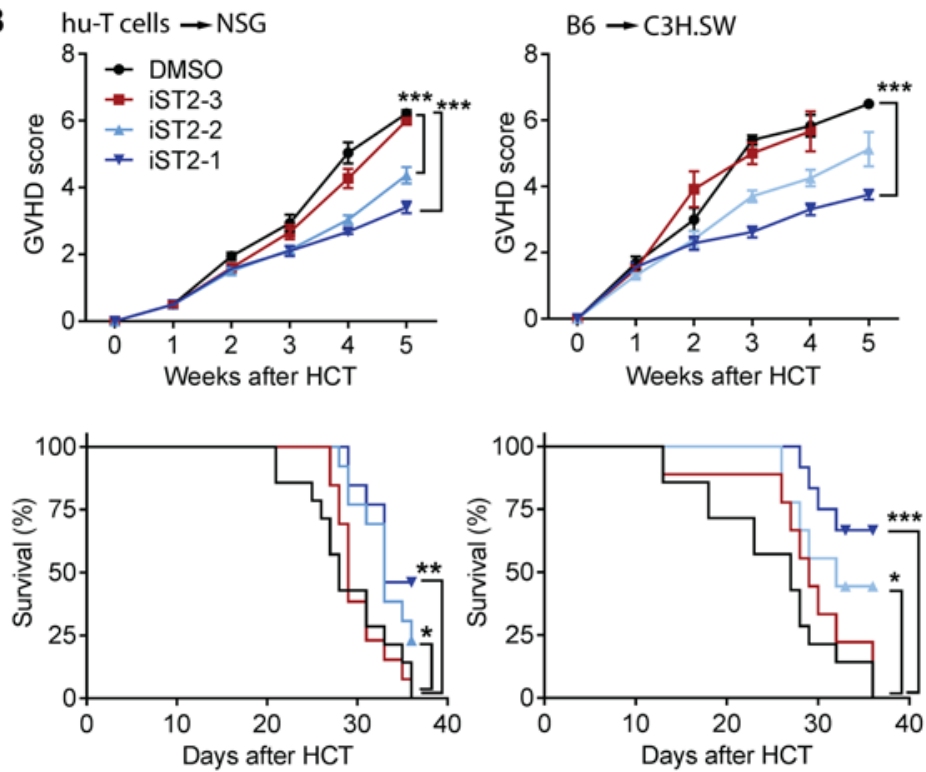

Figure 5. SST2 and IFN- $\gamma$ levels, GVHD scores, and survival curves for GVHD disease model mice treated with ST2 inhibitors. (A) Plasma levels of human and murine sST2 and IFN- $\gamma$ in the hu-T cells $\rightarrow$ NSG and $\mathrm{B} 6 \rightarrow \mathrm{C} 3 \mathrm{H}$. SW GVHD models from day 7 to 28 . Data represent mean $\pm \operatorname{SEM}(n=3$ per group for NSG and $n=6$ for $\mathrm{C} 3 \mathrm{H}$. SW). (B) CVHD scores and survival curves for the hu-T cells $\rightarrow$ NSG and B6 $\rightarrow$ C3H.SW GVHD models. Data represent mean \pm SEM for GVHD scores, and Kaplan-Meier curves for survival ( $n=13$ per group). $P$ values were calculated for GVHD scores by unpaired $t$ test and for survival by log-rank test. ${ }^{*} P$ $<0.05 ;{ }^{* *} P<0.01$ ${ }^{* * *} P<0.001$.

present. Data from this second mouse GVHD model led us to further examine the influence of the most effective compound, iST2-1, on the GVL effects in the same B6 $\rightarrow$ C3H.SW GVHD model. We found that treatment with iST2-1 maintained the beneficial GVL activity in mice receiving HCT.

Although the effects of our ST2 inhibitors on the mouse GVHD models paralleled the findings in the study of the neutralizing human ST2 antibody treatment (34), higher dosages of ST2 inhibitors compared with the human ST2 antibody were required because of the weaker inhibitory activities of the ST2 inhibitors. Still, small molecules provide many attractive pharmacokinetic properties when considering clinical application for the treatment of different diseases. For example, the small-molecule CCR5 inhibitor, maraviroc, was used in GVHD prophylaxis to reduce lymphocyte migration in a recent phase $1 / 2$ trial (59), despite results showing that CCR5 antibodies were also effective in a mouse model of GVHD (60). Further, sST2 is also a biomarker of chronic GVHD (16) where small-molecule inhibitors can be an advantageous route for administration of a long-term medication. Currently, the benefits of iST2-1 in GVHD management remain limited by its potency; however, further optimization is feasible based on the structure-activity relationship of our select iST2-1 analogs. Our investigation of the potential binding sites of iST2-1 identified locations on ST2 that can be interrogated by single-site mutagenesis and the precise binding mode of iST2-1 with ST2 needs to be defined by crystal structure determination in future studies. Our computational binding site analysis indicated that S3r for $(R)$-iST2-1 and S3s for $(S)$-iST2-1 are in close proximity. An inhibitor design strategy, similar to bivalent inhibitors $(61,62)$, of connecting 2 isomers of iST2-1 using a linker group may lead to improved inhibitor potency. In addition, the efficacy of iST2-4, the third chemotype inhibitor, in the GVHD experimental models remains to be determined. 

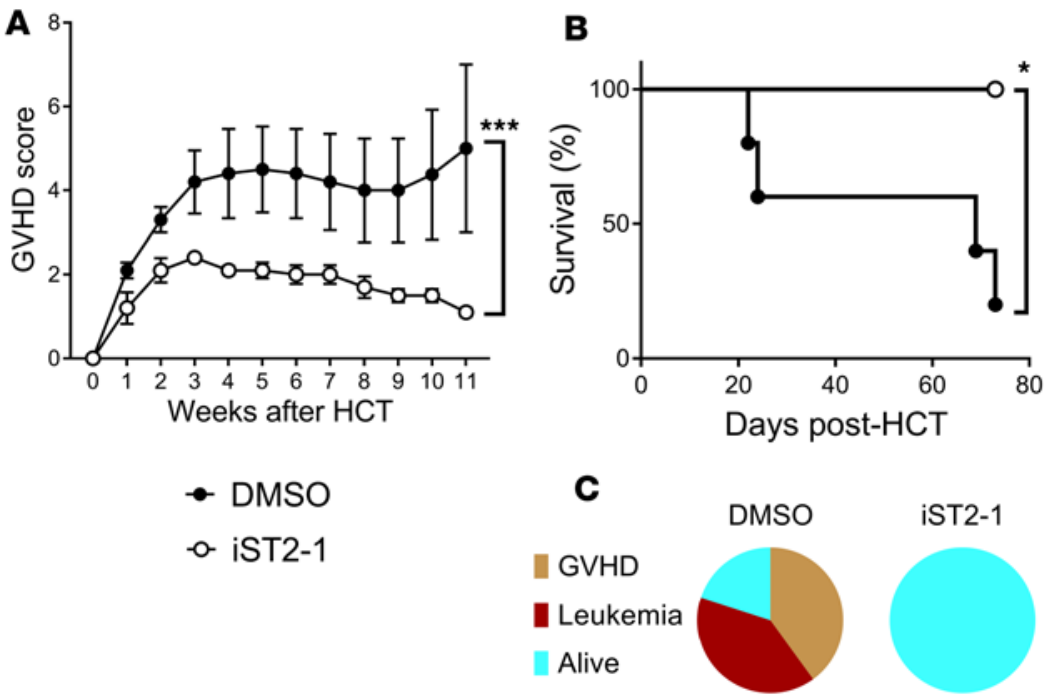

Figure 6. GVL activity in the C3H.SW mouse model treated with DMSO or iST2-1. (A) GVHD scores. Data represent mean \pm SEM, unpaired $t$ test, $P=0.0001$ ( $n=5$ per group), and (B) Kaplan-Meier survival curves of C3H.SW mice that received $2 \times 10^{4} \mathrm{GFP}^{+} \mathrm{MLL}-\mathrm{AF9}$ leukemia cells with allo-HCT (B6 $\rightarrow \mathrm{C3H} . \mathrm{SW}$ ) and were treated with DMSO control (filled circles) or iST2-1 (open circles), log-rank $P=0.013$ ( $n=5$ per group). The treatment schedule was the same as in Figure 4A. (C) Percentage of mice that succumbed to GVHD or leukemia or remained alive with treatment of DMSO or iST2-1 at the end of the study (day 73 ). ${ }^{*} P<0.05 ;{ }^{* *} P<0.001$.

Cytokine receptors are an important class of therapeutic targets; yet, only biologics are approved treatment options for targeting their ECDs that recognize specific cytokines. Small molecules inhibiting the interaction between cytokines and cytokine receptors have eluded identification because of their extensive protein-protein interaction interface. Our data suggest the feasibility of targeting $\mathrm{ST} 2^{\mathrm{ECD}}$ with small-molecule inhibitors to achieve in vivo activity in experimental GVHD models. Although our ST2 inhibitors are not highly selective between sST2 and ST2, they yielded decreased sST2 levels and lesser severity in in vivo GVHD models. In in vivo systems, soluble cytokine receptors can be locally produced at high concentrations and become systemic after entering the circulation. We have shown that the ratio of sST2 to ST2 is increased 40- to 70-fold in the intestine of the mouse GVHD model (34), and another study showed that a high local concentration of sST2 within the myocardium can be induced by myocardial pressure-overload to enhance hypertrophy in mice (63). We interpret the specific inhibition of sST2 observed in vivo as the action of ST2 inhibitors on a large excess amount of sST2 over ST2 expressed on T cells, similar to what we have found for inhibition with the anti-ST2 antibodies in the same GVHD model. Spatiotemporal changes in SST2 and ST2 expression in different cells and tissues in the GVHD progression and their contribution to T cell activation remain to be defined. Future in-depth studies of the mechanisms of action of our ST2 inhibitors in GVHD, including the consequences of their differential inhibition of ST2 and sST2, will provide insights for further studies of our ST2 inhibitors in other disease models (64-66) such as inflammatory bowel disease (67). Structurally, the ST2 transmembrane domain restrains the D3 domain of ST2 to limit the conformational motion of the D1 and D2 domains of ST2. In contrast, sST2, containing only the D1-D3 domains, possesses higher conformational flexibility (44) in blood circulation. To discover and develop sST2-selective inhibitors, differences in the conformational flexibility between ST2 and SST2 may offer opportunities.

Finally, the expanding roles of the ST2/IL-33 axis in type 2 diabetes and obesity (68), pulmonary arterial hypertension (69), and idiopathic pulmonary fibrosis as well as systemic sclerosis $(70,71)$ suggest that therapeutic development of ST2 inhibitors will have a broad impact on disease management beyond GVHD.

\section{Methods}

AlphaLISA. An AlphaLISA had been optimized in our pilot screening, including adjustment of several parameters in accordance with NIH recommendations (72) at the University of Michigan. From our pilot screening, the assay is robust with an $\mathrm{S} / \mathrm{B}$ of $=11, \mathrm{CV}=0.7 \%$, and $Z^{\prime}$ factor (73) of 0.95 . The assay was adapted for HTS in the Indiana University School of Medicine, Chemical Core Facility that has the capa- 
bility to optimize and automate biochemical-based assays for screening a large library of compounds with adequate diversity and representation of chemical space. The buffer used in the assay contains $14.37 \mathrm{ml}$ PBS, $30 \mu 1$ Tween 20 (Sigma-Aldrich), and $600 \mu 1$ of $5 \%$ bovine serum albumin (BSA, Gemini) in PBS. A mixture of ST2 and IL-33 was prepared by adding $0.30 \mu 1$ of $14.1 \mu \mathrm{M}$ recombinant human ST2-Fc Chimera (R\&D Systems, 523-ST) and $0.30 \mu 1$ of 55.25 biotinylated recombinant human IL-33 (United States Biologicals, I7663-97 with custom biotinylation) to $1.4 \mathrm{ml}$ of assay buffer. In the HTS, each well of a 384well Proxi-plate (PerkinElmer) was first blocked by $100 \mu 1$ of assay buffer for 1 hour at room temperature. After removing the blocking buffer, $20 \mu 1$ of the ST2/IL-33 mixture was pipetted into each well and incubated at room temperature for 1 hour followed by addition of compounds at $17 \mu \mathrm{M}(0.8 \mu \mathrm{l})$ to each well and incubated at room temperature for 1 hour. Ten microliters of $60 \mu \mathrm{g} / \mathrm{ml}$ anti- $6 \times$ His-conjugated AlphaLISA acceptor beads (PerkinElmer) were added to each well and incubated at room temperature for 1 hour before addition of $10 \mu \mathrm{l}$ of $60 \mu \mathrm{g} / \mathrm{ml}$ of streptavidin-labeled AlphaLISA donor beads (PerkinElmer) for incubation at room temperature for 30 minutes. Incubation of acceptor and receptor beads was conducted in darkness. The well with $3 \mathrm{nM}$ ST2 alone was used as a negative control, whereas the ST2/IL-33 mixture with a human ST2 antibody (R\&D Systems, MAB523) added at $0.45 \mathrm{ng} / \mu \mathrm{l}$ was used as the positive control. Plates were read using the Envision Plate Reader (PerkinElmer).

HEK-BLUE IL-33 assay. HEK-Blue IL-33 cells purchased from InvivoGen (catalog hkb-hil33) were designed to detect bioactive IL-33 by monitoring activation of the NF- $\mathrm{kB}$ and AP-1 pathways. They were generated by stable transfection of HEK-Blue cells with the IL1RL1 gene. These cells express a SEAP reporter gene under the control of the IFN- $\beta$ minimal promoter fused to $5 \mathrm{NF}-\mathrm{\kappa B}$ and $5 \mathrm{AP}-1$ binding sites. Stimulation of HEK-Blue IL-33 cells with human IL-33, but not with IL-18 or TNF- $\alpha$, activates the NF- $\mathrm{KB}$ and AP-1 pathways, triggering the production of SEAP. Levels of SEAP in the supernatant can be easily determined with QUANTI-Blue.

HEK-Blue IL-33 cells were grown at $37^{\circ} \mathrm{C}$ with $5 \% \mathrm{CO}_{2}$ in Dulbecco's modified Eagle's medium (DMEM) supplemented with $4.5 \mathrm{~g} / 1$ glucose, 10\% (vol/vol) fetal bovine serum (FBS), 2 mM L-glutamine, $50 \mathrm{U} / \mathrm{ml}$ penicillin, $50 \mu \mathrm{g} / \mathrm{ml}$ streptomycin, and $100 \mu \mathrm{g} / \mathrm{ml}$ Normocin. Suspended cells from preparations were then dispensed in a 96-well plate at $190 \mu 1 /$ well with a density of $265,000 \mathrm{cells} / \mathrm{ml}$ in each well. In the NF-kB activation assay, cells were treated with $10 \mu 1 \mathrm{IL}-33$ solution at $100 \mathrm{ng} / \mathrm{ml}$ and $50 \mu 1$ of serial dilutions of tested compounds or DMSO (positive control). For the negative control, $10 \mu 1$ of TNF- $\alpha$ solution at $100 \mathrm{ng} / \mathrm{ml}$ was used. The final volume in each well was $250 \mu 1$. After 20 hours of incubation in a $\mathrm{CO}_{2}$ incubator at $37^{\circ} \mathrm{C}, 160 \mu \mathrm{l}$ of the cell supernatants was mixed with $40 \mu 1$ of QUANTIBlue (InvivoGen) in each well for $1-3$ hours at $37^{\circ} \mathrm{C}$ before measurement of SEAP levels using a spectrophotometer at $620-655 \mathrm{~nm}$. Blockade of the stimulation of HEK-Blue IL-33 cells by recombinant human IL-33 was confirmed by using the neutralizing human ST2 antibody. The $\mathrm{IC}_{50}$ values of active compounds were calculated based on the percentage decreases in SEAP levels with serial dilutions relative to the signals obtained from the positive and negative controls.

$S A X S$ experiment. Procedures for expression and purification of recombinant human ST2 (HisTEV-ST2 ${ }^{\mathrm{ECD}}$, sequence: K19-P323) have been reported previously (44). The binding affinity between our recombinant human ST2 and human IL-33 was confirmed (44). The buffer used in ST2 is $10 \mathrm{mM}$ HEPES, pH 7.5, $150 \mathrm{mM} \mathrm{NaCl}, 4 \%$ glycerol. For ST2/iST2-1, $500 \mu \mathrm{M}$ racemic iST2-1 was added to ST2 giving a concentration of $4 \%$ DMSO in the final buffer. For all samples, ST2 was incubated with iST2-1 for 1 hour at $4^{\circ} \mathrm{C}$, purified through FPLC (Superdex 200), and then concentrated to $4 \mathrm{mg} / \mathrm{ml}$. Serial dilution of the samples was then performed to prepare 4 concentrations $(4,3,2,1 \mathrm{mg} / \mathrm{ml})$ of ST2 and ST2/iST2-1. The prepared samples were shipped to SIBYLS (74) overnight. SAXS data were collected via the mail-in program $(74,75)$ using the SIBYLS beamline (12.3.1) (74) at the Advanced Light Source in Lawrence Berkeley National Laboratory.

The 1-dimensional buffer-subtracted SAXS profile at each protein concentration was calculated from the average of 20 measurements in ST2 and ST2/iST2-1 using in-house python scripts. For each protein sample, the SAXS profiles at different protein concentrations were inspected to exclude contribution from protein aggregation (caused by cumulative radiation damage) before merging to one composite SAXS profile using the SCÅTTER program. Using the SCÅTTER program, we calculated the Kratky plot, $R_{g}$, maximum dimension $\left(D_{\max }\right)$, and $P(r)$. The ab initio shape function derived from the $P(r)$ was calculated by DAMMIF (76) first and then refined by DAMMIN (77) implemented in SCÅTTER using 13 runs to obtain the average solution. Although the gel data indicated the MW of our recombinant glycosylated human ST2 expressed in High Five cells is approximately $50 \mathrm{kDa}$, analysis of the SAXS profile gave an estimate of $42.4 \mathrm{kDa}$. 
Cluster analysis and the pharmacophore model. A total of 1,823 compounds with $30 \%$ or greater inhibition from the primary screening were included in the analysis. Each compound was annotated with a bitmap fingerprint consisting of 166 MACCS (41) keys. Similarity indexes between 2 compounds were assigned by the Tanimoto coefficient (78) calculated from their fingerprints. Cutoff values of 0.65 in similarity and overlap were used in the cluster analysis to classify compounds into structurally similar groups. A total of 1,260 groups consisting of 1-62 members were obtained. After the cluster analysis, we inspected each group to remove frequent binders to multiple bioassays identifiable in either PubChem or in our internal curated database. Compounds that contained substructures alerted in PAINS (42) were also excluded. The analysis led to selecting 45 representative compounds for the secondary dose-dependent confirmatory screening. The initial discovery study identified 4 confirmed hits (CD1959-0211, CB6114052, CB5107562, and NAT13-343201) that were used in the pharmacophore model construction. Among the 4 compounds, CD1959-0211 has 3 chiral centers. We have explored different stereoisomers when performing the structural alignment and selected the (3aS,4S,9bS)-8-chloro-3a,4,5,9b-tetrahydro-3H-cyclopenta[c]quinoline-4-carboxylic acid (shown in Supplemental Figure 1) to construct the pharmacophore model. All calculations and analyses were performed using the MOE program (79).

Hits enrichment based on pharmacophore searching and structure-based screening. To achieve diversity in hits enrichment without selecting an overwhelming number of compounds for validation, we selected a 4-feature pharmacophore model as a filter for searching for additional candidates from commercial compound libraries using the MOE program. Besides the ligand-based pharmacophore screening, we also used the crystal structure of ST2/IL-33 (PDBID: 4K3C) for structure-based screening. The ST2 structure was extracted from the crystal structure of ST2/IL-33 and prepared using the Maestro suite from Schrodinger (80). The targeted binding site was selected at the D2 domain of ST2 and a cavity between the D1 and D2 domains because mutational studies $(43,81)$ indicated their importance to the interaction between ST2 and IL-33. Default settings were chosen to generate the grids for performing structure-based screening of compound libraries using the XP docking mode in the Glide program (82) from the Maestro suite. We included 741 compounds of the azaindole library from Chem-X-Infinity in the structure-based screening and chose 20 compounds for validation. The focus library, evaluated in our experimental assays, consists of 118 compounds selected from the ligand-based pharmacophore search, analog searches from the vendors' online tools, and in silico structure-based screening.

Microsomal stability studies. We determined the metabolic stability of ST2 inhibitors using pooled CD-1 mouse liver microsomes (purchased from XenoTech). Each compound at $1 \mu \mathrm{M}$ was incubated with 0.5 $\mathrm{mg} / \mathrm{ml}$ microsomes and $1.7 \mathrm{mM}$ cofactor $\beta$-NADPH in $0.1 \mathrm{M}$ phosphate buffer $(\mathrm{pH}=7.4)$ containing 3.3 $\mathrm{mM} \mathrm{MgCl}{ }_{2}$ at $37^{\circ} \mathrm{C}$. The final solution contained less than $0.1 \%$ of DMSO. Forty microliters of reaction mixture was then taken out at 5, 10, 15, 30, 45, and 60 minutes of incubation and the enzymatic reaction of the mixture was stopped immediately by adding 3-fold excess cold acetonitrile containing $100 \mathrm{ng} / \mathrm{ml}$ of internal standard for quantification. Supernatant of the collected fractions was obtained via centrifugation at 21,130 $\mathrm{g}$ for 10 minutes and used for LC-MS/MS analysis. The amount of ST2 inhibitors not digested by the mouse liver microsome at each time point was then determined. We plotted the natural log of the amount of parent compound against time to calculate the half-life of each ST2 inhibitor.

$M L R$. Purified $\mathrm{CD}^{+}$and $\mathrm{CD} 8^{+} \mathrm{T}$ cells from PBMCs of one donor were stained with CFSE $(5 \mu \mathrm{M})$, washed twice, and cultured with irradiated T cell-depleted PBMCs from another donor for 7 days in the presence of DMSO as control or ST2 inhibitors at different concentrations in RPMI supplemented with 10\% FBS, $2 \mathrm{mM}$ L-glutamine, $1 \%$ penicillin/streptomycin, $1 \mathrm{mM}$ sodium pyruvate, and $50 \mu \mathrm{M} \beta$-mercaptoethanol (Life Technologies). Cells were collected and stained for flow cytometric analysis after 7 days of incubation. The ratio of responders to stimulators was 1:2 and no cytokines were added in the MLR.

Induction and assessment of GVHD. Mice underwent allo-HCT as previously described (34). Briefly, in miHA-mismatched GVHD models (B6 $\rightarrow$ C3H.SW), C3H.SW recipient mice first received 1,100 cGy TBI $\left({ }^{137} \mathrm{Cs}\right.$ source $)$ at day -1 . Then, recipient mice were injected intravenously with $\mathrm{T}$ cell-depleted BM cells $\left(5 \times 10^{6}\right)$ plus splenic T cells $\left(2 \times 10^{6}\right)$ from allogeneic donors at day 0 . T cells from donor mice were enriched by the murine CD90.2 microbead T Cell Isolation Kit (Miltenyi Biotec), and T cell-depleted BM was prepared with CD90.2 Microbeads (Miltenyi Biotec). In the xenogeneic GVHD model (human T cells $\rightarrow$ NSG mice), irradiated (300 cGy) NSG mice were transplanted with human total T cells from PBMCs $\left(1 \times 10^{6}\right)$ at day 0 . PBMCs were prepared from human peripheral blood Leukopacks from healthy donors that were purchased from the Central Indiana Blood Center under an IRB-approved protocol. PBMCs were 
isolated and drawn by Ficoll (GE Healthcare) density gradient centrifugation. Human total T cells were purified using the human CD4 and CD8 Microbeads Isolation Kit (Miltenyi Biotec). Mice were housed in sterilized micro-isolator cages and maintained on acidified water $(\mathrm{pH}<3)$ for 3 weeks. NSG mice were maintained on food supplemented with Uniprim and acidic water during the whole time course of the experiment. Survival was monitored daily. Clinical GVHD scores were assessed weekly as described previously (83). According to animal protocols approved by the IRB, mice were euthanized when the clinical score reached 6.5. Recipient mice in both models received twice daily i.p. injection of $200 \mu 1 \mathrm{PBS}$ with $0.5 \%$ DMSO as control or ST2 inhibitors for 21 days starting from day -1 to day 20 (iST2-1, $41.4 \mathrm{mg} / \mathrm{kg} /$ day; iST2-2, $18 \mathrm{mg} / \mathrm{kg} /$ day; and iST2-3, $20.5 \mathrm{mg} / \mathrm{kg}$ /day [in $200 \mu \mathrm{PBS}$ with $0.5 \%$ DMSO]). We followed the $\mathrm{NIH}$ guidelines for the care and use of laboratory animals in this study.

Isolation of intestinal cells. Single-cell suspensions were prepared from intestines as described previously (34). Briefly, intestines were flushed with PBS to remove fecal matter and mucus. Fragments $(<0.5 \mathrm{~cm})$ of intestines were digested in $10 \mathrm{ml}$ DMEM containing collagenase type B ( $2 \mathrm{mg} / \mathrm{ml}$; Roche), deoxyribonuclease I (10 $\mu \mathrm{g} / \mathrm{ml}$; Roche), and $4 \%$ BSA (Sigma-Aldrich) at $37^{\circ} \mathrm{C}$ with shaking for 90 minutes. The digested mixture was then diluted with $30 \mathrm{ml}$ plain DMEM, filtered through a 70- $\mu \mathrm{m}$ strainer, and centrifuged at $850 \mathrm{~g}$ for 10 minutes. The cell pellets were suspended in $5 \mathrm{ml}$ of $80 \%$ Percoll (GE Healthcare), overlaid with $8 \mathrm{ml}$ of $40 \%$ Percoll, and spun at $800 \mathrm{~g}$ for 20 minutes at $4^{\circ} \mathrm{C}$ without braking. Enriched lymphocytes were collected from the interface. For sorting of intestinal stem cells, we did not perform Percoll separation.

Enzyme-linked immunosorbent assay (ELISA). Plasma samples were collected from mice with GVHD treated with ST2 inhibitors or DMSO control. Every 7 days, human IFN- $\gamma$ and human sST2 levels were measured from plasma of NSG mice that received human T cells, whereas murine IFN- $\gamma$ and murine sST2 levels were measured in C3H.SW mice that received C57BL/6 BM and T cells. Levels of human and murine IFN- $\gamma$ were measured using the DuoSet ELISA Kit (R\&D Systems); levels of human and murine sST2 were measured using the Quantikine ELISA Kit (R\&D Systems).

Flow cytometric analysis. Cells from MLR in vitro or isolated from intestine ex vivo were washed and preincubated with purified anti-mouse CD16/CD32 mAb for $10-20$ minutes at $4^{\circ} \mathrm{C}$ to prevent nonspecific binding of the antibodies. The cells were subsequently incubated for 30 minutes at $4^{\circ} \mathrm{C}$ with antibodies for surface staining. Fixable viability dye was used to distinguish live cells from dead cells. A FoxP3/Transcription Factor Staining Buffer Set and Fixation and Permeabilization Kit were used for intracellular transcription factor and cytokine staining. For cytokine staining, cells were restimulated with phorbol myristate acetate (PMA; 50 $\mathrm{ng} / \mathrm{ml})$, ionomycin ( $1 \mu \mathrm{g} / \mathrm{ml}$; Sigma-Aldrich), and brefeldin A for 4-6 hours prior to any staining. All human and murine antibodies used in this work were obtained from eBioscience as shown in Supplemental Table 2.

Induction and assessment of GVL effect. C3H.SW mice were lethally irradiated (1,100 cGy) 1 day before $\mathrm{BM}$ transplantation. Recipient mice were injected intravenously with $5 \times 10^{6} \mathrm{~B} 6 \mathrm{BM}$ cells and $2 \times 10^{6}$ enriched B6 splenic T cells and $2 \times 10^{4}$ MLL-AF9 from C3H.SW background cells on day -1 . Mice were treated intraperitoneally with iST2-1 or DMSO control at the same dosage and dosing schedule as described for GVHD experiments. Mice were monitored daily for survival and leukemia development and weekly for GVHD score assessment. We attributed death to leukemia based on a high percentage of $\mathrm{eGFP}^{+}$cells and death to GVHD only if the mice had a low percentage of $\mathrm{GFP}^{+}$cells and a high GVHD score.

Statistics. Data are shown as either means \pm standard deviation (SD) from a single representative experiment with triplicates or means \pm standard error of the mean (SEM) from at least 2 independent experiments shown as error bars. The statistical comparisons between the treatment and control groups were made using unpaired $t$ tests. For 3 groups or more, each subplot involved 2 group-wise comparisons. Prior to analysis, the normality assumption was examined. All tests were 2-sided at the significance level of 0.05 . All calculations were conducted using the GraphPad Prism program version 7. A $P$ value less than 0.05 was considered significant.

Study approval. Animal experiments and euthanasia protocols performed in this study were approved by the Indiana University School of Medicine institutional animal care committee (IACUC protocol 11000).

\section{Author contributions}

AMR, ED, JCR, KC, JZ, ZB, AR, and ML performed experiments. AMR, ED, JCR, GLH, BG, ML, and JAS analyzed the data. CYY and SP designed the experiments, analyzed the data, wrote the manuscript, and supervised the project. 


\section{Acknowledgments}

We acknowledge the HTS center at Indiana University for collection of data from the compound screening. We thank Kathryn Burnett for collecting the SAXS data through the SIBYLS mail-in program using Advanced Light Source beamline 12.3.1.2 (to C.Y. Yang). SAXS data collection at SIBYLS was funded by the DOE-BER IDAT grant (DE-AC02-05CH11231) and NIGMS-supported ALS-ENABLE (P30 GM124169-01). We also thank Sherry Wang and Xinquan Wang for suggestions on protein expression and SAXS experiments and Shaomeng Wang for providing computing resources for this work. This work was supported by the NIH/National Cancer Institute (R01 CA174667 to C.Y. Yang and S. Paczesny), the Leukemia and Lymphoma Society (grant 1293-12 to S. Paczesny), a Fast Forward Medical Innovation Award from the University of Michigan (to C.Y. Yang), and the NIH though the University of Michigan (P30 CA046592) by use of the following Rogel Cancer Center Core: Center of Structural Biology and Center for Chemical Genomics and Pharmacokinetics and Mass Spectrometry Core.

Address correspondence to: Chao-Yie Yang, University of Michigan, B520-1230A, NCRC, 1600 Huron Parkway, Ann Arbor, Michigan 48105, USA. Phone: 734.764.2449; Email: chaoyie@umich.edu. Or to: Sophie Paczesny, Indiana University School of Medicine, 1044 W. Walnut Street, Room R4-425, Indianapolis, Indiana 46202, USA. Phone: 317.278.5487; Email: sophpacz@iu.edu.

1. Kotas ME, Medzhitov R. Homeostasis, inflammation, and disease susceptibility. Cell. 2015;160(5):816-827.

2. Netea MG, et al. A guiding map for inflammation. Nat Immunol. 2017;18(8):826-831.

3. Mantovani A, Locati M, Vecchi A, Sozzani S, Allavena P. Decoy receptors: a strategy to regulate inflammatory cytokines and chemokines. Trends Immunol. 2001;22(6):328-336.

4. Levine SJ. Molecular mechanisms of soluble cytokine receptor generation. J Biol Chem. 2008;283(21):14177-14181

5. Heaney ML, Golde DW. Soluble receptors in human disease. J Leukoc Biol. 1998;64(2):135-146.

6. Chalaris A, Garbers C, Rabe B, Rose-John S, Scheller J. The soluble interleukin 6 receptor: generation and role in inflammation and cancer. Eur J Cell Biol. 2011;90(6-7):484-494.

7. Meijers WC, van der Velde AR, de Boer RA. ST2 and galectin-3: Ready for prime time? EJIFCC. 2016;27(3):238-252.

8. Seo DH, et al. Interleukin-33 regulates intestinal inflammation by modulating macrophages in inflammatory bowel disease. Sci Rep. 2017;7(1):851.

9. Parikh RH, Seliger SL, Christenson R, Gottdiener JS, Psaty BM, deFilippi CR. Soluble ST2 for prediction of heart failure and cardiovascular death in an elderly, community-dwelling population. J Am Heart Assoc. 2016;5(8):e003188.

10. Pascual-Figal DA, et al. Soluble ST2 is a marker for acute cardiac allograft rejection. Ann Thorac Surg. 2011;92(6):2118-2124.

11. Díaz-Jiménez D, et al. Soluble ST2: a new and promising activity marker in ulcerative colitis. World J Gastroenterol. 2011;17(17):2181-2190.

12. Vander Lugt MT, et al. ST2 as a marker for risk of therapy-resistant graft-versus-host disease and death. $N$ Engl J Med. 2013;369(6):529-539.

13. Kanakry CG, et al. Single-agent GVHD prophylaxis with posttransplantation cyclophosphamide after myeloablative, HLAmatched BMT for AML, ALL, and MDS. Blood. 2014;124(25):3817-3827.

14. Rotz SJ, Dandoy CE, Davies SM. ST2 and endothelial injury as a link between GVHD and microangiopathy. $N$ Engl J Med 2017;376(12):1189-1190.

15. Hartwell MJ, et al. An early-biomarker algorithm predicts lethal graft-versus-host disease and survival. JCI Insight. 2017;2(3):e89798.

16. Yu J, et al. Biomarker panel for chronic graft-versus-host disease. J Clin Oncol. 2016;34(22):2583-2590.

17. McDonald GB, Tabellini L, Storer BE, Lawler RL, Martin PJ, Hansen JA. Plasma biomarkers of acute GVHD and nonrelapse mortality: predictive value of measurements before GVHD onset and treatment. Blood. 2015;126(1):113-120.

18. Ponce DM, et al. High day 28 ST2 levels predict for acute graft-versus-host disease and transplant-related mortality after cord blood transplantation. Blood. 2015;125(1):199-205.

19. Abu Zaid M, et al. Plasma biomarkers of risk for death in a multicenter phase 3 trial with uniform transplant characteristics post-allogeneic HCT. Blood. 2017;129(2):162-170.

20. Levine JE, et al. A prognostic score for acute graft-versus-host disease based on biomarkers: a multicentre study. Lancet Haematol. 2015;2(1):e21-e29.

21. Schmitz J, et al. IL-33, an interleukin-1-like cytokine that signals via the IL-1 receptor-related protein ST2 and induces T helper type 2-associated cytokines. Immunity. 2005;23(5):479-490.

22. Tominaga S. A putative protein of a growth specific cDNA from BALB/c-3T3 cells is highly similar to the extracellular portion of mouse interleukin 1 receptor. FEBS Lett. 1989;258(2):301-304

23. Tominaga S, Tago K, Tsuda H, Komine M. Dual function of IL-33 on proliferation of NIH-3T3 cells. Cytokine. 2015;72(1):105-108

24. Garlanda C, Dinarello CA, Mantovani A. The interleukin-1 family: back to the future. Immunity. 2013;39(6):1003-1018.

25. Löhning M, et al. T1/ST2 is preferentially expressed on murine Th2 cells, independent of interleukin 4, interleukin 5, and interleukin 10, and important for Th2 effector function. Proc Natl Acad Sci USA. 1998;95(12):6930-6935.

26. Salimi M, et al. A role for IL-25 and IL-33-driven type-2 innate lymphoid cells in atopic dermatitis. J Exp Med. 2013;210(13):2939-2950.

27. Schmitz J, et al. IL-33, an interleukin-1-like cytokine that signals via the IL-1 receptor-related protein ST2 and induces T helper 
type 2-associated cytokines. Immunity. 2005;23(5):479-490.

28. Endo Y, et al. The interleukin-33-p38 kinase axis confers memory T helper 2 cell pathogenicity in the airway. Immunity. 2015;42(2):294-308

29. Günther S, et al. IL-1 family cytokines use distinct molecular mechanisms to signal through their shared co-receptor. Immunity. 2017;47(3):510-523.e4.

30. Guo L, et al. IL-1 family members and STAT activators induce cytokine production by Th2, Th17, and Th1 cells. Proc Natl Acad Sci USA. 2009;106(32):13463-13468.

31. Blom L, Poulsen BC, Jensen BM, Hansen A, Poulsen LK. IL-33 induces IL-9 production in human CD4 ${ }^{+}$T cells and basophils PLoS One. 2011;6(7):e21695.

32. Wynn TA. Type 2 cytokines: mechanisms and therapeutic strategies. Nat Rev Immunol. 2015;15(5):271-282

33. Schiering C, et al. The alarmin IL-33 promotes regulatory T-cell function in the intestine. Nature. 2014;513(7519):564-568

34. Zhang J, et al. ST2 blockade reduces sST2-producing T cells while maintaining protective mST2-expressing T cells during graftversus-host disease. Sci Transl Med. 2015;7(308):308ra160.

35. Hayakawa H, Hayakawa M, Kume A, Tominaga S. Soluble ST2 blocks interleukin-33 signaling in allergic airway inflammation. J Biol Chem. 2007;282(36):26369-26380.

36. Ferrara JL, Levine JE, Reddy P, Holler E. Graft-versus-host disease. Lancet. 2009;373(9674):1550-1561.

37. Griesenauer B, Paczesny S. The ST2/IL-33 axis in immune cells during inflammatory diseases. Front Immunol. $2017 ; 8: 475$.

38. Jones SA, Rose-John S. The role of soluble receptors in cytokine biology: the agonistic properties of the sIL-6R/IL-6 complex. Biochim Biophys Acta. 2002;1592(3):251-263.

39. Schreiber G, Walter MR. Cytokine-receptor interactions as drug targets. Curr Opin Chem Biol. 2010;14(4):511-519.

40. Kakkar R, Lee RT. The IL-33/ST2 pathway: therapeutic target and novel biomarker. Nat Rev Drug Discov. 2008;7(10):827-840

41. Durant JL, Leland BA, Henry DR, Nourse JG. Reoptimization of MDL keys for use in drug discovery. J Chem Inf Comput Sci. 2002;42(6):1273-1280

42. Baell JB, Holloway GA. New substructure filters for removal of pan assay interference compounds (PAINS) from screening libraries and for their exclusion in bioassays. JMed Chem. 2010;53(7):2719-2740.

43. Liu X, et al. Structural insights into the interaction of IL-33 with its receptors. Proc Natl Acad Sci USA. 2013;110(37):14918-14923

44. Yang CY, et al. Conformational sampling and binding site assessment of suppression of tumorigenicity 2 ectodomain. PLoS One. 2016;11(1):e0146522.

45. Yang CY. Identification of potential small molecule allosteric modulator sites on IL-1R1 ectodomain using accelerated conformational sampling method. PLoS One. 2015;10(2):e0118671.

46. Rambo RP, Tainer JA. Characterizing flexible and intrinsically unstructured biological macromolecules by SAS using the Porod-Debye law. Biopolymers. 2011;95(8):559-571.

47. Putnam CD, Hammel M, Hura GL, Tainer JA. X-ray solution scattering (SAXS) combined with crystallography and computation: defining accurate macromolecular structures, conformations and assemblies in solution. Q Rev Biophys. 2007;40(3):191-285.

48. Yang CY, Wang S. Hydrophobic binding hot spots of Bcl-xL protein-protein interfaces by cosolvent molecular dynamics simulation. ACS Med Chem Lett. 2011;2(4):280-284.

49. Buch I, Giorgino T, De Fabritiis G. Complete reconstruction of an enzyme-inhibitor binding process by molecular dynamics simulations. Proc Natl Acad Sci USA. 2011;108(25):10184-10189.

50. Mehrotra A, Leventhal J, Purroy C, Cravedi P. Monitoring T cell alloreactivity. Transplant Rev (Orlando). 2015;29(2):53-59.

51. Fanning LR, et al. Allogeneic transplantation of multiple umbilical cord blood units in adults: role of pretransplant-mixed lymphocyte reaction to predict host-vs-graft rejection. Leukemia. 2008;22(9):1786-1790.

52. Paczesny S, et al. A biomarker panel for acute graft-versus-host disease. Blood. 2009;113(2):273-278.

53. Paczesny S, et al. Elafin is a biomarker of graft-versus-host disease of the skin. Sci Transl Med. 2010;2(13):13ra2

54. Ferrara JL, et al. Regenerating islet-derived 3-alpha is a biomarker of gastrointestinal graft-versus-host disease. Blood. 2011;118(25):6702-6708.

55. Tomuleasa C, et al. MicroRNAs as biomarkers for graft-versus-host disease following allogeneic stem cell transplantation. Ann Hematol. 2015;94(7):1081-1092.

56. Jamani K, et al. Prognosis of grade 3-4 acute GVHD continues to be dismal. Bone Marrow Transplant. 2013;48(10):1359-1361.

57. Antin JH. Steroid-refractory acute graft-versus-host disease: is there an effective therapy? Biol Blood Marrow Transplant. 2014;20(2):146-148

58. Kumar S, Minnich MD, Young PR. ST2/T1 protein functionally binds to two secreted proteins from Balb/c 3T3 and human umbilical vein endothelial cells but does not bind interleukin 1. J Biol Chem. 1995;270(46):27905-27913.

59. Moy RH, et al. Clinical and immunologic impact of CCR5 blockade in graft-versus-host disease prophylaxis. Blood. 2017;129(7):906-916

60. Murai M, et al. Active participation of CCR5(+)CD8(+) T lymphocytes in the pathogenesis of liver injury in graft-versus-host disease. J Clin Invest. 1999;104(1):49-57.

61. Kitov PI, Shimizu H, Homans SW, Bundle DR. Optimization of tether length in nonglycosidically linked bivalent ligands that target sites 2 and 1 of a Shiga-like toxin. J Am Chem Soc. 2003;125(11):3284-3294.

62. Mack ET, et al. Dependence of avidity on linker length for a bivalent ligand-bivalent receptor model system. J Am Chem Soc. 2012;134(1):333-345.

63. Chen WY, Hong J, Gannon J, Kakkar R, Lee RT. Myocardial pressure overload induces systemic inflammation through endothelial cell IL-33. Proc Natl Acad Sci USA. 2015;112(23):7249-7254.

64. De la Fuente M, MacDonald TT, Hermoso MA. The IL-33/ST2 axis: Role in health and disease. Cytokine Growth Factor Rev. 2015;26(6):615-623

65. Liew FY, Girard JP, Turnquist HR. Interleukin-33 in health and disease. Nat Rev Immunol. 2016;16(11):676-689

66. Mathews LR, et al. Elevated ST2 distinguishes incidences of pediatric heart and small bowel transplant rejection. Am J Transplant. 2016;16(3):938-950.

67. Salas A. The IL-33/ST2 axis: yet another therapeutic target in inflammatory bowel disease? Gut. 2013;62(10):1392-1393. 
68. Vasanthakumar A, et al. The transcriptional regulators IRF4, BATF and IL-33 orchestrate development and maintenance of adipose tissue-resident regulatory T cells. Nat Immunol. 2015;16(3):276-285.

69. Rhodes CJ, et al. Plasma proteome analysis in patients with pulmonary arterial hypertension: an observational cohort study. Lancet Respir Med. 2017;5(9):717-726.

70. Rice LM, et al. A proteome-derived longitudinal pharmacodynamic biomarker for diffuse systemic sclerosis skin. J Invest Dermatol. 2017;137(1):62-70.

71. Wagner A, Köhm M, Nordin A, Svenungsson E, Pfeilschifter JM, Radeke HH. Increased serum levels of the IL-33 neutralizing sST2 in limited cutaneous systemic sclerosis. Scand J Immunol. 2015;82(3):269-274.

72. Inglese J, et al. High-throughput screening assays for the identification of chemical probes. Nat Chem Biol. 2007;3(8):466-479.

73. Zhang JH, Chung TD, Oldenburg KR. A simple statistical parameter for use in evaluation and validation of high throughput screening assays. J Biomol Screen. 1999;4(2):67-73.

74. Classen S, et al. Implementation and performance of SIBYLS: a dual endstation small-angle X-ray scattering and macromolecular crystallography beamline at the Advanced Light Source. J Appl Crystallogr. 2013;46(Pt 1):1-13.

75. Hura GL, et al. Robust, high-throughput solution structural analyses by small angle X-ray scattering (SAXS). Nat Methods. 2009;6(8):606-612.

76. Franke D, Svergun DI. DAMMIF, a program for rapid ab-initio shape determination in small-angle scattering. $J$ Appl Crystallogr. 2009;42(Pt 2):342-346.

77. Svergun DI. Restoring low resolution structure of biological macromolecules from solution scattering using simulated annealing. Biophys J. 1999;76(6):2879-2886.

78. Rogers DJ, Tanimoto TT. A computer program for classifying plants. Science. 1960;132(3434):1115-1118.

79. MOE: Molecular Operating Environment. Chemical Computing Group. http://www.chemcomp.com. Accessed July 17, 2018.

80. Friesner RA, et al. Glide: a new approach for rapid, accurate docking and scoring. 1. Method and assessment of docking accuracy. J Med Chem. 2004;47(7):1739-1749.

81. Lingel A, et al. Structure of IL-33 and its interaction with the ST2 and IL-1RAcP receptors--insight into heterotrimeric IL-1 signaling complexes. Structure. 2009;17(10):1398-1410.

82. Friesner RA, et al. Extra precision glide: docking and scoring incorporating a model of hydrophobic enclosure for proteinligand complexes. J Med Chem. 2006;49(21):6177-6196.

83. Li W, et al. Proteomics analysis reveals a Th17-prone cell population in presymptomatic graft-versus-host disease. JCI Insight. 2016;1(6):e86660 